\title{
A comprehensive map of the dendritic cell transcriptional network engaged upon innate sensing of HIV
}

Jarrod S. Johnson ${ }^{1,2, \mp, *}$, Nicholas De Veaux ${ }^{3, \mp}$, Alexander W. Rives $^{3}$, Xavier Lahaye ${ }^{4}$, Sasha Y. Lucas $^{2}$, Brieuc Pérot ${ }^{5}$, Marine Luka ${ }^{5}$, Lynn M. Amon², Aaron Watters ${ }^{3}$, Alan Aderem ${ }^{2,6}$, Nicolas Manel $^{4}$, Dan R. Littman ${ }^{7,8}$, Richard Bonneau ${ }^{3,9,10}$, and Mickaël M. Ménager ${ }^{5,7,11, *}$

${ }^{1}$ University of Utah, Department of Biochemistry, Salt Lake City, UT, USA 84112

${ }^{2}$ Center for Infectious Disease Research, Seattle, WA 98109, USA

${ }^{3}$ Flatiron Institute, Center for Computational Biology, Simons Foundation, New York, NY 10010, USA.

${ }^{4}$ Immunity and Cancer Department, Institut Curie, PSL Research University, INSERM U932, 75005 Paris, France

${ }^{5}$ Laboratory of Inflammatory Responses and Transcriptomic Networks in Diseases, Imagine Institute, INSERM UMR 1163, ATIP-Avenir team, Université Paris Descartes Sorbonne Paris Cité, 24 boulevard du Montparnasse, 75015, Paris, France

${ }^{6}$ Department of Immunology, University of Washington School of Medicine, Seattle, WA 98109, USA

${ }^{7}$ The Kimmel Center for Biology and Medicine of the Skirball Institute, New York University School of Medicine, New York, NY 10016, USA

${ }^{8}$ Howard Hughes Medical Institute, New York University School of Medicine, New York, NY 10016, USA

${ }^{9}$ Department of Biology, Center for Genomics and Systems Biology, New York University, New York, NY 10003, USA.

${ }^{10}$ Center for Data Science, New York University, New York, NY 10011, USA.

${ }^{\mp}$ Authors contributed equally

${ }^{\star}$ Correspondence: mickael.menager@institutimagine.org (MMM)

${ }^{*}$ Correspondence: jarrod.johnson@biochem.utah.edu (JSJ)

${ }^{11}$ Lead Contact 


\section{Graphical Abstract}

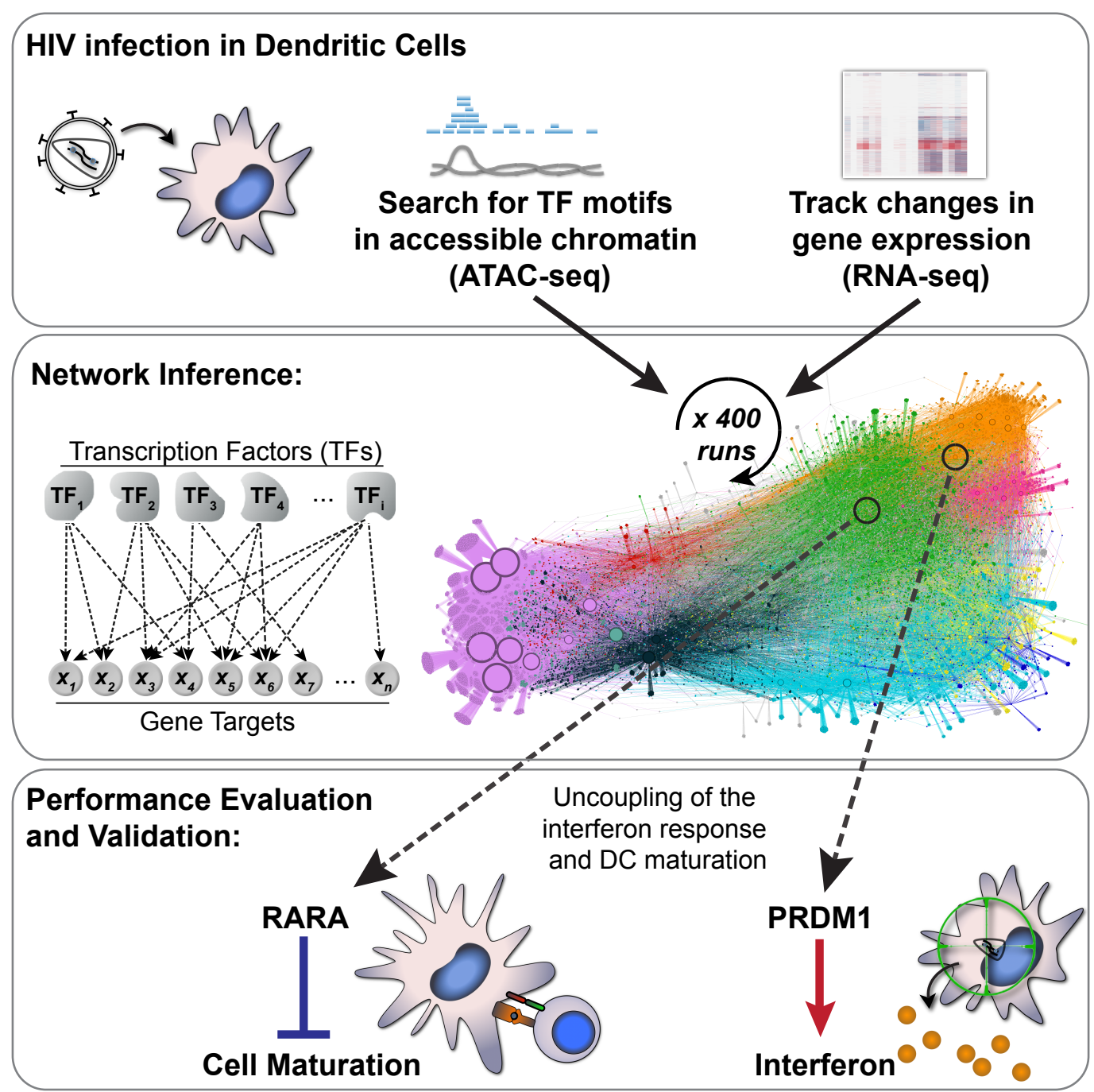




\section{Summary}

Transcriptional programming of the innate immune response is pivotal for host protection. However, the transcriptional mechanisms that link pathogen sensing with innate activation remain poorly understood. During infection with HIV-1, human dendritic cells (DCs) can detect the virus through an innate sensing pathway leading to antiviral interferon and DC maturation. Here, we developed an iterative experimental and computational approach to map the innate response circuitry during HIV-1 infection. By integrating genome-wide chromatin accessibility with expression kinetics, we inferred a gene regulatory network that links 542 transcription factors with 21,862 target genes. We observed that an interferon response is required, yet insufficient to drive DC maturation, and identified PRDM1 and RARA as essential regulators of the interferon response and DC maturation, respectively. Our work provides a resource for interrogation of regulators of HIV replication and innate immunity, highlighting complexity and cooperativity in the regulatory circuit controlling the DC response to infection.

\section{Key Words}

network inference, DNA sensing, innate signaling, cGAS, STING, IRF3, NF-кB, chromatin modification, ATAC-seq, RNA-seq 


\section{Introduction}

The host's ability to rapidly alter gene expression in order to defend against infection is a central element of the innate immune response. Host-encoded pattern recognition receptors (PRRs) detect components of foreign microorganisms and self-derived immunostimulatory products (Cao, 2016; Ivashkiv and Donlin, 2014; Iwasaki and Medzhitov, 2015). When a pathogen is sensed, PRRs initiate signal transduction cascades that lead to activation of multiple transcription factors (TFs), which subsequently rewire gene expression to protect the host. Considering that aberrations in innate immunity are hallmarks of many disorders, including chronic viral diseases, neurodegeneration, diabetes, and cancer (Corrales et al., 2017; Heneka et al., 2014; Wada and Makino, 2016), it is not surprising that transcriptional activation of innate immune signaling is under tight control, with the goal of maintaining a sensitive response to infectious threats while avoiding unwanted inflammation and autoimmunity. In the case of HIV-1 infection, however, innate immune responses are insufficient for host protection and become dysregulated during progression to AIDS (Fernandez et al., 2011; Sandler et al., 2014; Schoggins et al., 2011).

Dendritic cells (DCs) serve key functions in host defense and are among the first cells thought to contact HIV-1 during transmission (lijima et al., 2008). Myeloid DCs express an arsenal of PRRs and link innate detection of microbes to activation of pathogen-specific adaptive immune responses (Banchereau et al., 2000; Thery and Amigorena, 2001). These cells express cell surface receptors for HIV-1 entry, but the virus undergoes limited productive infection in DCs and does not trigger robust immune responses due to the presence of restriction factors (Granelli-Piperno et al., 2004; Manel et al., 2010; Smed-Sorensen et al., 2005). The primary restriction factor in myeloid DCs is SAMHD1, an enzyme that exhibits phosphohydrolase activity and depletes the cellular pool of dNTPs required for HIV reverse transcription (Hrecka et al., 2011; Laguette et al., 2011). This restriction can be overcome if DCs are first exposed to virus-like particles that deliver the lentiviral accessory protein, Vpx (absent 
in HIV-1 but encoded by SIV and HIV-2) (Goujon et al., 2006; Mangeot et al., 2000). Vpx targets SAMHD1 for degradation, enabling productive HIV-1 infection, sensing of viral components, and activation of innate immune responses (Manel et al., 2010).

Innate immune responses against HIV-1 are triggered in myeloid DCs by the sensor cyclic GMP-AMP synthase (cGAS), which detects reverse-transcribed HIV cDNA in a process that requires concomitant HIV capsid protein interaction with the cellular protein NONO, and is facilitated by other proximal factors (Gao et al., 2013; Jonsson et al., 2017; Lahaye et al., 2018; Yoh et al., 2015). Downstream of innate sensing initiated by cGAS, several transcription factors are activated, including IRF and NF-kB family members, which drive induction of interferons (IFNs), IFN-stimulated genes (ISGs), and inflammatory cytokines, and promote DCs to transition from an inactive immature state to a mature, activated state. In addition to upregulating innate antiviral factors, mature DCs express at their cell surface the costimulatory factors CD80 and CD86, which are critical for programming adaptive responses (Goubau et al., 2013; Iwasaki, 2012). The DC transcriptional response circuitry involves feedback loops that engage multiple activator and repressor TFs that collectively influence thousands of gene targets during IFN signaling and DC maturation (Ivashkiv and Donlin, 2014). For these reasons it is difficult to understand the constellation of TF-target gene connections that operate during innate immune responses using traditional approaches.

Work from our groups and others has demonstrated that gene regulatory network inference, when applied to study dynamic systems such as macrophage activation, Th17 lymphocyte polarization, and the innate immune response to cytosolic DNA, has predicted the functions of key transcriptional regulators whose involvement was previously unknown (Ciofani et al., 2012; Gilchrist et al., 2006; Lee et al., 2013; Ramsey et al., 2008). Our earlier computational methods pioneered the use of time series perturbations and the incorporation of structured "prior information" into gene regulatory network inference (Bonneau et al., 2006; Greenfield et al., 2013; Greenfield et al., 2010; Madar et al., 2010). In this report we have 
demonstrated that network inference is improved by ensemble-learning across hundreds of individual computational runs, with each run predicated on subsampled information in the "prior" network. We have integrated chromatin accessibility data together with genome-wide measurements of gene expression to infer and experimentally validate a network describing the human dendritic cell transcriptional circuitry that is engaged upon HIV sensing.

\section{Results}

\section{Perturbation of human DCs in face of diverse innate immune and viral stimuli}

With the goal of better understanding how DCs respond to innate immune stimuli, we exposed immature monocyte-derived DCs to a battery of innate immune agonists and viral challenges, generating data-sets comprised of RNA-sequencing (RNA-seq) and assays for transposase-accessible chromatin (ATAC-seq) (Figure 1A; Table S1). We tracked the kinetics of gene expression and DC maturation during HIV-1 infection compared to classic innate agonists by infecting DCs with a single-cycle HIV-1 reporter virus (HIV-1-GFP), transducing with a non-replicating lentivirus (LKO-GFP), or stimulating in parallel with the TLR4 agonist, lipopolysaccharide (LPS) or the double-stranded RNA mimetic, polyinosinic:polycytidylic acid (pIC) for 2, 8, 24, and $48 \mathrm{~h}$. LPS and pIC triggered rapid changes in gene expression in human DCs across several gene clusters (Figure $1 \mathrm{~B}$ and Table S2), resembling what has been reported for mouse bone marrow-derived DCs (Amit et al., 2009; Chevrier et al., 2011). Similarly, infection with HIV-1-GFP led to induction of innate immune genes (Figure 1B; clusters $5 \& 8$ ), but did so with delayed kinetics compared to LPS and pIC, likely due to time-dependent accumulation of reverse transcription products, integration, and virus replication progressing over the first 24 h (Gao et al., 2013; Johnson et al., 2018). Gene expression profiles were consistent with the timing of DC maturation as scored by flow cytometry (Figure S1A), with LPS and pIC stimulation leading to early and robust induction of CD86. DCs infected with HIV-1-GFP 
did not mature until $48 \mathrm{~h}$, and only minimally responded to LKO-GFP (Figure S1A), as we previously described (Johnson et al., 2018; Manel et al., 2010).

In agreement with the earlier analyses, we observed that RNA-seq samples from infected and uninfected DCs could be clearly distinguished when visualized by Principal Component Analysis (PCA) (Figure S1B). Samples at 24 and $48 \mathrm{~h}$ time points could be further separated when grouped specifically by time or cell sorting condition: GFP-negative (HIVexposed but not expressing GFP), GFP-positive (HIV-infected), HIV-CD86-low (HIV-infected, immature DC), and HIV-CD86-high (HIV-infected, mature DC) (Figure S1C). We also used gene set enrichment analysis (GSEA) (Subramanian et al., 2005) to evaluate the qualitative nature of the innate response and uncovered strong associations between HIV-infected samples and gene sets for IFN alpha/beta signaling, inflammatory signaling, and DC maturation (full GSEA results available in supplemental materials). The most significant enrichment was found with the Hallmark IFN Response (Figure 1C), which peaked at $24 \mathrm{~h}$ post infection. Canonical ISGs were highly upregulated during HIV-1-GFP infection (Figure 1D) and their induction correlated with activation of IFNB1 and IFNL1 (Figure S1D). Having characterized the DC transcriptional response to HIV-1 infection, which included known maturation and interferon response signatures, we next sought, to identify regions of open chromatin that may be accessible to TF binding in order to define possible TF-to-gene target relationships that regulate the innate response.

\section{ATAC-seq reveals time-dependent chromatin opening at innate immune gene promoters}

Analyzing genome-wide chromatin accessibility represents a powerful way to assess the presence of regulatory elements such as promoters and enhancers in mammalian cells (Buenrostro et al., 2013; Johnson et al., 2018). To match the experimental conditions used for RNA-seq, we profiled changes in open chromatin by performing ATAC-seq on DCs that were mock treated or infected with HIV-1-GFP for 2, 8, 24, and $48 \mathrm{~h}$, and sorted by flow cytometry 
(Figure 2A-B). We identified 88k high-confidence peaks across all ATAC-seq conditions (Table S3). Similar to the RNA-seq samples, the ATAC-seq samples for mock and HIV-1-GFP could be separated by PCA through time and status of infection with minimal variation between donors (Figure S2A-B). By plotting genome-wide changes in gene expression together with changes in chromatin accessibility at transcription start sites, we observed that global changes in chromatin accessibility are temporally linked with stages of HIV-1 single-round infection and DC maturation (Figure 2C). At $24 \mathrm{~h}$ post infection, we found that the induction of IFNB1, IFNL1, and most Hallmark IFN response genes in GFP-negative and GFP-positive populations were linked with increases in chromatin accessibility. By $48 \mathrm{~h}$, expression intensity and chromatin accessibility of these genes began to subside, with particularly noticeable reductions in chromatin accessibility for IFNB1 and IFNL1 to levels below baseline.

To investigate changes in chromatin accessibility in more detail, we plotted promoterassociated ATAC-seq peak height across the time series for two housekeeping control genes (PGK1 \& TBP), the interferon genes IFNB1 and IFNL1, well-defined ISGs, and IRF and NF-KB family members. Maximum chromatin accessibility for IFNB1, IFNL1 and several ISGs was detected at $24 \mathrm{~h}$ post infection in cells expressing the GFP reporter (Figures 2D; S2C-D). For IFN-related genes and the NF-KB family members, RELA, RELB, REL, and the related TF, HIVEP1, chromatin accessibility was higher in HIV-infected, CD86-low DCs that were not fully mature as compared to CD86-high, mature DCs (Figure 2D), suggesting that the chromatin state is linked to DC maturation status.

To determine whether mapping open chromatin using ATAC-seq in HIV-infected DCs offered specific advantages over publicly available datasets, we compared our ATAC-seq peaks to steady-state open chromatin data from CD14+ monocytes (Figure S2C-D). Several ISGs displayed high chromatin accessibility at baseline (STAT1, STAT2, IRF1, IRF7, IRF9, OASL, HLA-C, and ISG20), with peaks detected in both DC ATAC-seq samples and CD14+ monocyte data available from ENCODE (Figure S2C). HIV-1-GFP infection led to a transient increase in 
chromatin accessibility at ISG promoters that corresponded with known binding sites for IRF3, STAT1, and NF-KB (RELA). In contrast, IFNB1, IFNL1, and other ISGs (CXCL10, CXCL11, ISG15, LY6E, USP18, and IFIT1), displayed open chromatin in DCs infected with HIV-1-GFP, but not in mock-treated cells or in CD14+ monocytes (Figure S2D), supporting the use of celland condition-specific ATAC-seq data as a basis for network inference.

\section{Inferred network of DC transcriptomic changes following innate immune responses.}

To infer a predictive gene regulatory network we adapted our previously published algorithm (the Inferelator), which was designed to learn from mixes of steady state and dynamic data (Bonneau et al., 2006). The method can incorporate multiple data types to influence network model selection, including TF occupancy, cooperativity, and transcriptional profiling in response to innate immune stimuli (Figures $1 \& 2$ ). High-throughput methods like ATAC-seq and ChIP-seq can be used to guide network inference by defining "prior" information on network architecture, dramatically improving network model selection and predictive power (Arrieta-Ortiz et al., 2015; Miraldi et al., 2019; Siahpirani and Roy, 2017). Key to this study, we extended our previously published computational methods for learning the regulation of gene networks through the Inferelator (Arrieta-Ortiz et al., 2015; Bonneau et al., 2006; Ciofani et al., 2012; Greenfield et al., 2010; Madar et al., 2010) by integrating results obtained from RNA-seq and ATAC-Seq experiments performed at the bulk level in a time-course fashion (Figure 3A).

Here, prior information on network architecture is derived from the $88 \mathrm{k}$ peaks of chromatin accessibility data generated by our ATAC-seq experiments that tracked DC responses to HIV infection (Figures $2 \mathrm{~A} \& 3 \mathrm{~A}$ ). Using curated TF binding motif databases, we established possible TF-gene target relationships by searching ATAC-seq peaks for TF motifs that we located within or up to $1 \mathrm{~kb}$ upstream of gene bodies (Figure $3 \mathrm{~B}$ ). This information was used to build a prior matrix that connected each gene with its possible TF regulators (Table S4). We then estimated TF activity over the course of stimulation based on the combined expression 
of a TF's predicted gene targets (Figure 3C). This step approximates the effect of unmeasured parameters, such as post-translational regulation and protein-protein interactions, on TF activity in a condition-dependent manner. We were thus able to model changes in TF activity that are semi-independent of changes in TF expression, as observed for IRF3 (Figure S3A, S3B). IRF3 activity was predicted to increase in response to LPS, pIC and HIV sensing, which are all known to drive IFN production through IRF3 phosphorylation. Our inference model also predicted that activity and expression for STAT2, IRF7, and RELA correlated with innate stimulation, as expected, given their well-defined roles in the innate response (Cao, 2016).

Once TF activity was estimated, the network structure prior was then used to bias model selection of TF-to-gene target regulatory interactions towards edges with prior information during network inference (Figure 3D). To improve the overall performance and stability of network inference, several aspects of the method (and key inputs) were tested, such as: different sources of priors (publicly available ENCODE data vs our ATAC-seq data), different TF binding motif databases (HOCOMOCO (Kulakovskiy et al., 2013) vs CisBp 2.0 (Weirauch et al., 2014)), and different model selection methods (Bayesian Best Subset Regression (BBSR) vs Elastic Net (EN)). Additionally, since every computational run is subject to stochasticity in the inference procedure, we evaluated whether network performance could be improved by combining hundreds of individual computation runs (selecting model components such as TF activity estimates that were stable across random subsamples of the structure prior; see STAR Methods). To evaluate the performance of networks inferred using the parameters described above, we used area under precision-recall curves (Madar et al., 2010) to compare the prediction and ranking of our TF-to-gene target regulatory edges against TRRUSTV2, a goldstandard reference database of human transcriptional regulatory events (Han et al., 2018), and well-known lists of ISGs (Kane et al., 2016; Schoggins et al., 2011; Shaw et al., 2017). We found that the EN regression model outperformed BBSR when ATAC-seq based networks were benchmarked against TRRUSTV2 (Figures 3E-F). We further improved precision-recall on 
TRRUST and known ISGs by bootstrapping 400 individual EN-ATAC inference runs into a converged network (EN-ATAC x400) (Figures 3E-H; S3C-D). This final EN-ATAC network correctly predicted 90 out of 97 "core" mammalian ISGs (Shaw et al., 2017) to be downstream of IRF and NF-KB family members (Figure S3E) and emphasized a role for IRF3 (Figure S3F). Thus, we were able to integrate gene expression and chromatin accessibility data to estimate a network with 542 TFs and 21,862 target genes, explaining $>2 / 3$ of the variance in our expression dataset (Table S5).

\section{Network clustering, differential gene expression analysis, and TF enrichment tests define key subregulatory groups.}

We have used this DC transcriptional response network to group the expression changes observed into sets of co-regulated genes with high confidence regulatory subnetworks. We first applied a cutoff to visualize the top $75 \mathrm{k}$ regulatory edges that displayed the highest confidence beta scores (Figure 4A) and then partitioned the network using Louvain-modularity clustering into 10 major "neighborhoods" of TF and target gene communities, with each cluster encompassing at least $1 \%$ of the total number of genes in the network (DC network topology can be freely explored using Gephi software by downloading the Gephi-formatted supplemental file, available in the STAR Methods section). We were able to assign putative biological functions to 7 out of 10 clusters based on pathway enrichment scores (see STAR Methods). The most striking enrichment scores were observed in the top right shoulder of the network, demarcating clusters that are predicted to function in the interferon response (Cluster 5 , IRFs \& Interferon), inflammation and cytokine production (Cluster 8, NF-kB \& Inflammation), and in response to xenobiotic stress (Cluster 2, Regulation of Cell Activation) (Figure 4B-D).

Activation of IRF, STAT, and NF-KB family members is centrally linked to interferon signaling during the innate immune response (Cao, 2016; Ivashkiv and Donlin, 2014). Fittingly, we found that several IRF (IRFs 1, 2, 3, 5, 7, 8, and 9), STAT1 \& STAT2, and all five NF-KB 
(RELA, RELB, REL, NFKB1, and NFKB2) populated the upper right shoulder of the network (Figure S4A), consistent with their overlap in motif preferences (Figure S4B). Interestingly, IRF4 and IRF5, which also have GAA- and GAAA- rich motif features similar to that of other IRFs (Figure S4C), are found positioned in different areas of the network. IRF4, in particular, is localized to the extreme lower left (Figure S4A; Cluster 1, Chromatin Modifiers) in proximity to pioneer factors and repressors that target large numbers of genes (Jankowski et al., 2016).

We next sought to establish the relative impact of each TF in the network during an innate response. Towards this end we performed hypergeometric tests to assess TF enrichment, and found high enrichment for IRF, STAT, and NF-KB family members during stimulation with HIV-1-GFP, LPS, and pIC (Figure S4D; Table S6), with kinetics closely matching what was observed for IFN production and DC maturation (Figures S1A, S1D). We also found mild enrichment of these TFs during LKO-GFP infection, supporting the concept that non-replicating lentiviral vectors are partially, if inefficiently, sensed by the innate immune system (Figure S4D) (Johnson et al., 2018). By highlighting network topology where genes are differentially expressed during treatment with HIV-1-GFP, LKO-GFP, LPS, and pIC, we determined that the majority of the transcriptional response is concentrated in Clusters 2,5 , and 8 (Regulation of Cell Activation, IRFs \& Interferon, and NF-KB \& Inflammation) (Figure S4E).

\section{Exploration and validation of modulators of the interferon response}

The first wave of the antiviral response is driven by production of type I and type III IFNs (Levy et al., 2011). In DCs the major type I and type III IFNs that are expressed are IFNB1 and IFNL1, respectively (Figure S1D). To visualize all high-confidence edges for IFNB1, IFNL1, and their regulators that were predicted by the network, we generated a subnetwork visualization tool (Figure 5A; see STAR Methods for instructions on how to access the Jupyter subnetwork widget). Upstream of both IFNB1 and IFNL1, we identified IRF3 as well as additional IRFs that have been reported to contribute to their induction under various conditions (such as IRF1, 
IRF5, IRF7, and IRF8) (Ivashkiv and Donlin, 2014)). Several unexpected factors were also predicted to be upstream regulators of IFNB1 and IFNL1 (Figure 5A), and of these TFs, we note that HIVEP1, CBFB, STAT2, PRDM1, and KLF13 were expressed at relatively high levels in DCs and in some cases exhibited dynamic expression changes during the innate response (Figure 5B).

We next sought to empirically test the function of several of these TFs in DCs, noting that PRDM1 and HIVEP1 were among the highest ranking TFs according to hypergeometric tests (Figure S4D). Using shRNA vectors we targeted these TFs for knockdown in DCs along side key sensors of HIV (cGAS and NONO) and the essential TF, IRF3. Among other potential TF candidates, we additionally tested KLF13, based on its relatively high expression and known role in recruiting coactivators p300/CBP and PCAF to drive expression of CCL5 (Ahn et al., 2007). We confirmed knockdown of these targets either by immunoblot ((Figure 5C) or qPCR (Figure S5A), and as expected, knockdown of cGAS and NONO potently inhibited DC maturation during HIV-1-GFP infection (Johnson et al., 2018; Lahaye et al., 2018; Lahaye et al., 2013) (Figures 5D-F; S5B-E). The most effective shRNA clones for HIVEP1, KLF13, and PRDM1 had no effect on viral infection. Under these conditions, we could not conclude whether HIVEP1 knockdown impacted DC maturation (Figure S5D). Knockdown of KLF13 partially inhibited DC maturation, which was significant for two shRNAs at high HIV-1-GFP MOI (Figure S5E). Moreover, we found that greater KLF13 knockdown correlated with lower levels of CD86 and inhibition of IFNL1 expression during HIV-1-GFP infection (Figure S5F). In a more dramatic way, knockdown of PRDM1 significantly inhibited CD86 induction and IFNL1, and these effects were observed with three independent shRNAs (Figure 5F-G). Together, these data suggest that while cellular factors such as cGAS, NONO, and IRF3 function as critical toggle switches during the innate response, additional TFs such as KLF13, and, to a greater extent, PRDM1, may operate to fine-tune the magnitude of the innate response. Furthermore, other TFs with high enrichment scores identified from experiments with wild type HIV-1, HIV-2, recombinant 
interferon, and discrete innate immune stimuli (Figure S5G; Table S6) warrant further exploration.

\section{Mature and immature DC populations cannot be distinguished based on IRF/STAT enrichment alone}

IRF and STAT activation is critical for inducing an interferon gene signature, but neutralization of IFN signaling does not completely block upregulation of inflammatory cell markers during HIV infection (Lahaye et al., 2013; Manel et al., 2010), suggesting that other factors in addition to these TFs contribute to DC maturation. Gene set enrichment analysis on CD86-low and CD86-high sorted DCs (Figures S1A; S2B) identified "DC Maturation" genes as significantly enriched in CD86-high compared to CD86-low populations (Figure 6A-C). However, we found no statistically significant difference in "Hallmark IFN Response" genes, with ISG expression levels and chromatin accessibility elevated in both conditions. (Figure 6B and S6AB). Thus, an IFN response is not sufficient to explain the differences in DC maturation status following HIV infection.

Consistent with our "DC Maturation" gene set results, the NF-kB family members (RELB, REL, NFKB1, RELA, and NFKB2) showed stronger enrichment in CD86-high than in CD86-low populations (Figure 6D-F) as did additional, unexpected, TFs (CLOCK, THRB, SRF, and HIVEP1) found in clusters 2 (Regulation of Cell Activation) and 8 (NF-kB \& Inflammation). Interestingly, pathway analysis of differentially accessible chromatin in CD86-high vs CD86-low conditions suggested that Retinoic Acid Receptor Alpha (RARA), PPARD, NCOR1, and the canonical NF-KB family member RELA (among other TFs) influenced the transition between immature and mature DCs (Figure S6C-E). When differentially expressed genes were assessed in CD86-high vs CD86-low conditions in similar fashion, pathway analysis predicted strong enrichment of protein-protein interactions with the RARA partner molecule, RXRA, pathways associated with nuclear receptor transcription, MyD88 signaling, and gene expression changes 
resembling published datasets from RARA perturbation (highest among other inflammatory activators) (Figures 6G-I). These analyses implicated RARA/RXRA nuclear hormone receptor signaling to be involved in regulating DC maturation.

\section{Identification of RARA as a critical TF for controlling DC maturation}

At steady-state, RARs bind retinoic acid response elements (RAREs) together with RXRs in a complex with nuclear corepressors (NCOR1/2) and suppress transcription of RAREdependent genes (Cunningham and Duester, 2015). Ligand binding leads to the dissociation of NCOR1/2 from RAR receptors and the recruitment of coactivators, which then drive gene expression. Given that RARA occupies a central region of Cluster 2 (Regulation of Cell Activation) (Figures 4D and $6 \mathrm{~J}$ ) and was predicted to influence DC maturation, we sought to assess its network connections and function compared to STAT2 and cGAS. Subnetwork visualization of RARA and close network edges displayed many connections between Cluster 2 (Regulation of Cell Activation) and Clusters 5 and 8 (IRF/Interferon and NF-KB/Inflammation, respectively) (Figure 7A). Many, but not all of these connections were detected using the Search Tool for the Retrieval of Interacting Genes (STRING) database (Szklarczyk et al., 2015), highlighting the position of RARA in close proximity to TFs involved in controlling cell fate, lipid \& sterol metabolism, interferon signaling, and inflammation (Figure 7B).

Knockdown of cGAS or STAT2 led to a pronounced loss of DC maturation during infection with HIV-1-GFP (Figure 7C-D). In contrast, knockdown of RARA upregulated CD86 at baseline and potentiated DC maturation during infection, suggesting RARA acts as a negative regulator of inflammation. Consistent with this notion, expression levels of many predicted targets of RARA increased in response to LPS and pIC treatment and to a lesser degree in response to HIV-1-GFP (Figure S7A). Moreover, we found an inverse relationship between gene expression of RARA and costimulatory molecules $C D 80$ and CD86 (Figure S7B), supported by the negative correlation between the estimated transcriptional activity of RARA 
and its measured gene expression (Figure S7C). Knockdown of RARA was associated with a slight but statistically significant decrease in production of interferon and interferon stimulated genes ISG15 and CXCL10 (Figure 7E and S7E-H), leading us to consider that loss of RARA might impact the balance between antiviral interferon responses and inflammatory responses that benefit virus replication. In agreement with this idea, we observed heightened virus transcription in RARA knockdown conditions (measured by GFP reporter expression, Figures 7C; 7E), as increased inflammation is a correlate of increased HIV replication (Deeks et al., 2013).

Direct modulation of RARA using the pharmacological agonist Am80 (Delescluse et al., 1991) triggered increased CD86 surface expression both at baseline and following HIV-1-GFP infection (Figure 7F-G). We also observed that treatment with Am80 or the classic RARA ligand, all-trans retinoic acid (ATRA), increased gene expression of CD86 (Figure S7D). Conversely, use of the RARA antagonist BMS 195614 reduced upregulation of CD86 during virus infection (Figure $7 \mathrm{H}-\mathrm{I}$ ). Although our findings from RARA agonists and antagonists may appear inconsistent with that of RARA knockdown, they are in line with the dual functions of nuclear receptors in recruiting either coactivators or corepressors of transcription, which will be further discussed below. Together, these data support our network predictions that spotlight a role for RARA in the regulation of DC maturation.

\section{Discussion}

Multiple transcription factors (TFs) are known to drive the innate response downstream of pathogen sensing in myeloid DCs (Ivashkiv and Donlin, 2014), but it remains unclear how production of interferon, differential expression of thousands of host genes, and DC maturation are coordinately regulated across the genome in response to HIV detection. To address this 
issue, we have employed a systems biology approach to understand how DCs respond to stimulation, generating steady state and time series RNA-seq under a battery of viral and innate immune perturbations combined with ATAC-seq measurements of chromatin accessibility (Figure 1A). We inferred a transcriptomic network linking 542 TFs to 21,862 targets and report $500 \mathrm{k}$ predicted regulatory interactions. To date, this represents one of the largest gene regulatory networks that has been charted in mammalian cells.

Computational strategies that combine ATAC-seq and gene expression data have recently been employed to identify key regulators of tissue-resident memory CD8+ T cells (Milner et al., 2017), define TF specificity across cell types (Cusanovich et al., 2018), and chart the landscape of human cancers (Corces et al., 2018). Here, we improved the stability of network inference by performing ensembles of analyses, with each run subsampling information from a structured network "prior" that was generated from our ATAC-seq data. By modeling changes in gene expression to be a function of combined TF activities, we reduced the complexity of multiple factors known to influence gene expression into a single parameter (such as changes in TF expression, post-translational modifications, and epigenetic marks on target genes). In doing so, our network revealed strong enrichment of TFs known to regulate the innate response, notably including IRF3, a key factor that is activated by phosphorylation and, as it is constitutively expressed, is not often identified in pathway analyses that emphasize changes in TF expression (Amit et al., 2009).

Our final gene regulatory network exhibited a high level of precision/recall against TFs targeting known ISGs and correctly predicted prominent roles for IRF-, STAT-, and NF-KB family members in the IFN response. We recovered 90 out of 97 "core" mammalian ISGs in the top $75 \mathrm{k}$ network edges as targets of IRFs, and note that the remaining 7 core ISGs were predicted to be downstream of STAT- and AP-1 family TFs (Table S5). Interestingly, of the 22 different IFN species measured by RNA-seq, we found IFNL1 to be upregulated more than any type I IFN, including IFNB1, during stimulation with LPS, pIC, or HIV-1. We know IFN signaling can 
provide both beneficial and detrimental effects during progression to AIDS (Sandler et al., 2014), yet the contribution of type-I vs type-III IFN in an in vivo response to HIV remains to be determined.

Among several TFs predicted to be upstream of IFN genes in our network, we found that PRDM1 (also known as Positive Regulatory Domain-I, PR/SET Domain-1, and BLIMP1) is an essential positive regulator of innate immune activation in response to HIV-1 infection in DCs. For almost 30 years, PRDM1 has been known to bind IFN promoter sequences (Keller and Maniatis, 1991). Since then, reports have suggested that PRDM1 is important for differentiation of plasma cells, is a risk factor for autoimmune disease, and can act to either positively or negatively regulate cell-specific cytokine production (Bonelt et al., 2019; Ko et al., 2018; Nutt et al., 2007). Here, we have revealed that PRDM1 promotes IFNL1 expression and DC maturation during HIV infection, in accordance with its high hypergeometric enrichment and cluster association with key IRFs in the network. Future exploration of our network could help uncover whether factors associated with PRDM1 determine how PRDM1 operates to promote or inhibit IFN production in a cell-specific manner.

By analyzing RNA-sequencing data from sorted cell populations, we have determined that induction of a robust ISG signature is insufficient for DC maturation, suggesting that factors in addition to PRDM1, IRF-, and STAT- family TFs members influence the transition from immature to mature DCs (Figure 6). In support of this notion, we have demonstrated that the retinoic acid receptor, RARA, acts as a negative regulator of DC maturation at basal state (Figures 7 \& S7). Based on our network analysis predicting that RARA and NF-KB activities govern the transition from immature to mature DC, together with our data indicating that RARA modulates innate immune responses, we propose a model in which, at basal state, RXR/RARA heterodimers bind to RAREs and inhibit DC maturation driven by NF-kB family members (Figure S7I). Perturbation of RARA expression, either by shRNA or unknown factors during the innate response relieves this inhibition and permits DC maturation (Figure S7J). Binding of 
ligand/retinoids to RARA results in the exchange of bound nuclear corepressors with nuclear coactivators that will also permit DC maturation. Thus, RARA knockdown and administration of RARA agonists can both achieve a similar biological end, albeit through different means (Figure S7K). In agreement with this model, using an RARA antagonist to block the release of nuclear corepressors inhibits DC maturation in response to HIV infection (Figure S7L). These results reinforce the reported involvement of retinoids in DC maturation (Geissmann et al., 2003; Szatmari and Nagy, 2008). Given that retinoic acid is known to activate MAPK phosphorylation and abrogate tolerance in a stressed intestinal environment (DePaolo et al., 2011), it is not unreasonable to envision that RARA activation might influence derepression of inflammatory gene expression during HIV infection.

Our model thus suggests that RARA function could provide another layer of control to govern DC maturation through sensing changes in metabolism. Differentiation of DCs in vitro and in vivo is known to depend on fatty acid synthesis (Rehman et al., 2013). In addition, in plasmacytoid DCs, lipid metabolism, RXR, and PPAR-related pathways can modulate type I IFN responses, suggesting that environmental cues such as retinoids help shape immune responses (Wu et al., 2016). It is tempting to draw parallels with how changes in fatty acid oxidation and cholesterol biosynthesis influence innate responses and determine cell fate (tolerogenic versus immunogenic DC) (Maldonado and von Andrian, 2010; York et al., 2015).

In addition to profiling how DCs respond to HIV, it is noteworthy to mention that we have profiled responses to a battery of classic innate immune stimuli, including LPS, pIC, cGAMP, R848, and recombinant interferon. This represents a valuable source of information that one can explore to compare similarities and differences among transcriptomic pathways bridging innate immune detection and production of inflammatory cytokines. We note that much of the transcriptomic activity observed for HIV infection in our network overlaps with that in response to LPS and pIC exposure; however, we found that HIV drives unique changes in fatty acid 
oxidation pathways (Johnson et al., 2018). These findings support the existence of an interplay between HIV infection, lipid metabolism, and retinoids that control IFN production and DC maturation, which will require further investigation.

Additionally, we note that HIV-1 infection drives transient changes in chromatin accessibility that likely precede or perhaps coincide with the induction and resolution phases of the innate response. Interestingly, we observed global changes in chromatin accessibility that were not always associated with changes in host gene expression. We speculate that this could reflect on either the nature of the innate response or on signals derived from events in the virus life cycle, as many of these changes were located in gene-dense regions known to be hot-spots for virus integration (Table S3, (Wang et al., 2007)).

Collective analyses of these data will help to further decipher the mechanisms of innate immune regulation that span initiation, feed-forward amplification, and the resolution of aberrant hyperactivation. Understanding DC maturation and IFN production will likely inform future antiviral strategies, since the robust activation of $\mathrm{T}$ cells, which is facilitated by mature DCs, is crucial for immunological control of HIV (Walker and McMichael, 2012). We anticipate that the quality of network inference will further improve as motif databases are refined and large-scale genetic perturbations become tractable in primary human DCs. The network presented herein highlights how the innate response depends on the coordinated action of multiple TFs and serves as a resource for further exploration of pathways that govern HIV replication and innate immunity. 


\section{Acknowledgments}

This project was supported the Howard Hughes Medical Institute (DRL); the Helen and Martin Kimmel Center for Biology and Medicine (DRL); by grants from the National Institutes of Health (F32AI093231 to JSJ; R21AI084633 to DRL; R01AI025032, R01AI032972, and U19AI100627 to AA); State funding from the Agence Nationale de la Recherche under "Investissements d'avenir" program (ANR-10-IAHU-01) (MMM) and ATIP-Avenir INSERM program (MMM).

\section{Author Contributions}

Conceptualization, JSJ, AR, NM, DRL, RB, and MMM; Methodology, JSJ, ND, AR, and MMM;

Software, ND, AR, AW; Formal Analysis, ND, AR, and LA; Investigation, JSJ, XL, SL, and

MMM; Writing - Original Draft, JSJ and MMM; Writing - Review \& Editing, all authors;

Resources, AA, NM, DRL, RB and MMM; Supervision, NM, AA, NM, DRL, RB, \& MMM;

Funding Acquisition, JSJ, AA, NM, DRL, RB, and MMM.

\section{Declaration of Interests}

The authors declare no competing interests. 
Figure 1

A

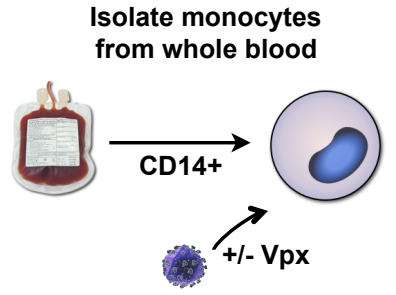

Perturb function with drugs \& genetic manipulation

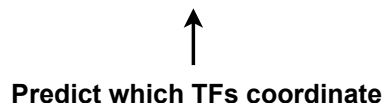

IFN and DC maturation

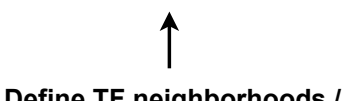

Functional clusters

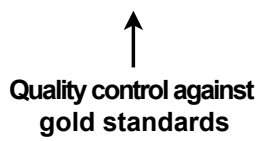

个
Derive DCs

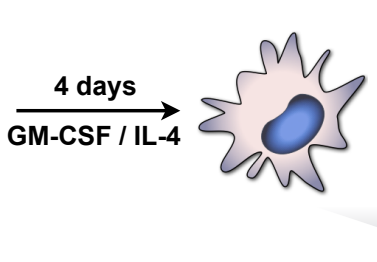

Stimulation

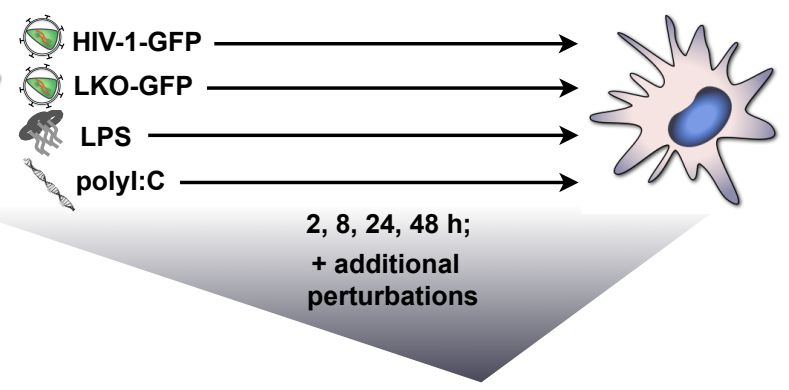

542 TFs

21,862 Target Genes

$\sim 75 \mathrm{k}$ Regulatory Interactions

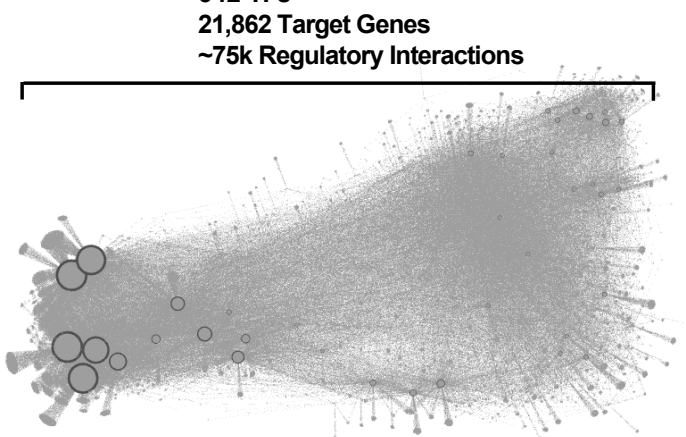

Visualize TF-target topology

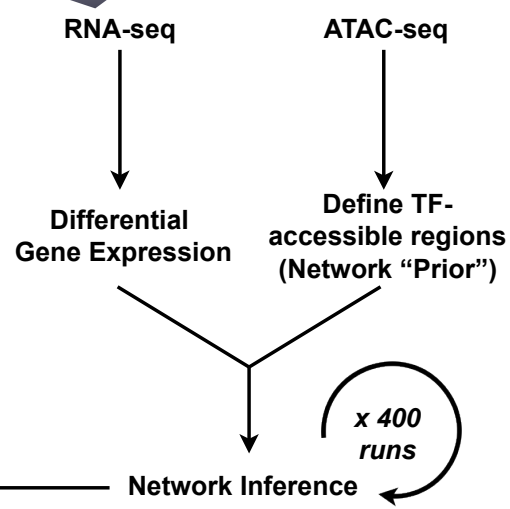

B

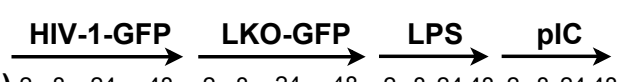

Time (h) $2 \quad 8 \underline{24} \stackrel{48}{\underline{4}} 28 \underline{24} \stackrel{48}{\underline{4}} 282448282448$ $\underset{G F P}{\text { GFP }}-\frac{24}{-++} \quad \frac{24}{-+}$

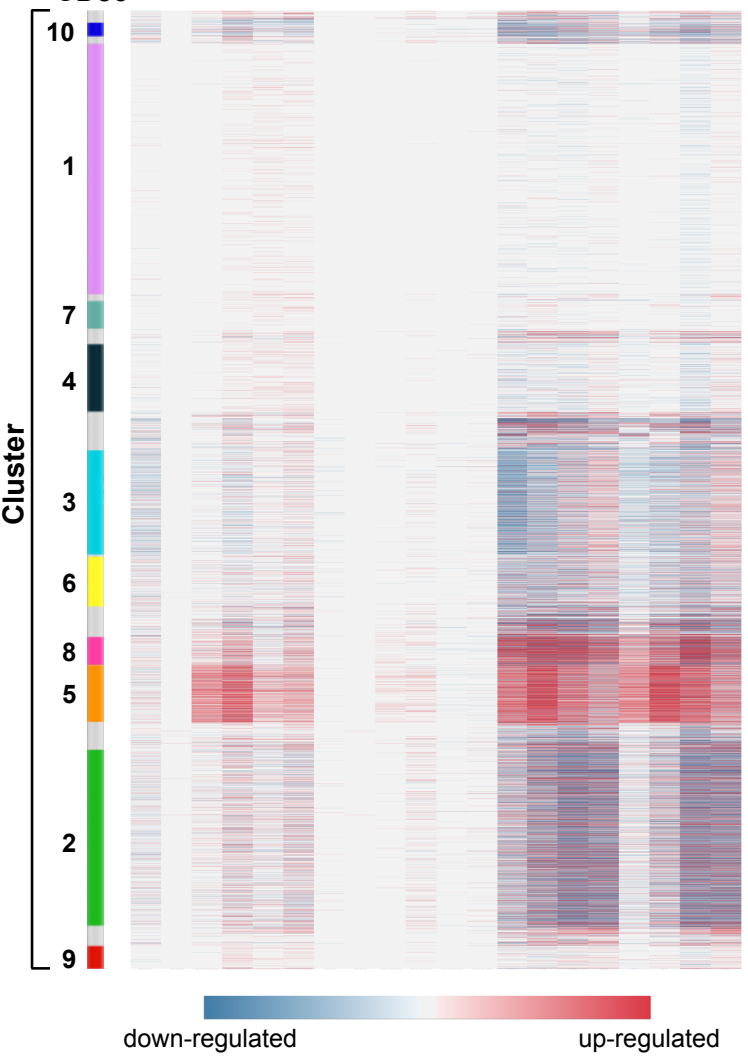

C Hallmark IFN Response:
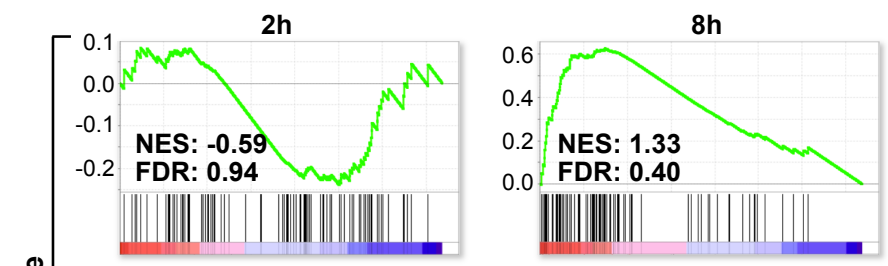

24h GFP-neg
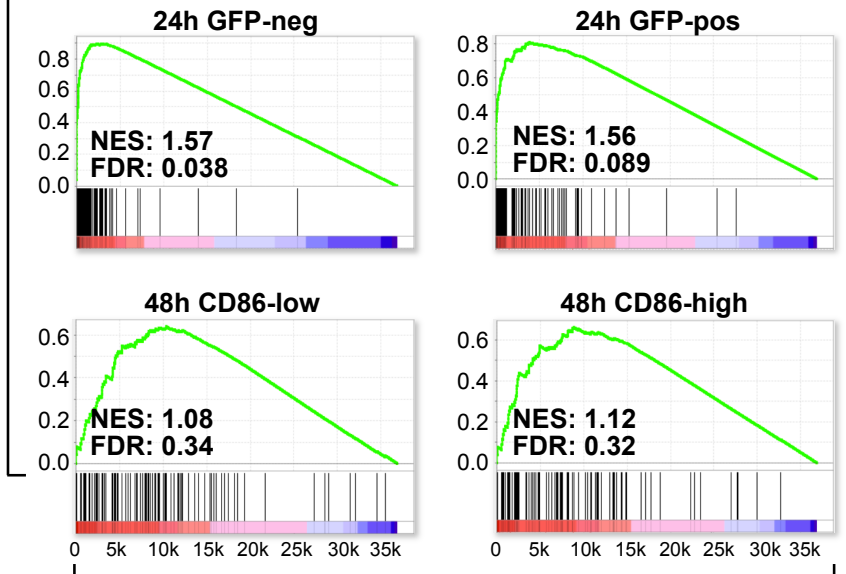

Rank in Dataset

D Top 25 Leading Edge Genes:

\begin{tabular}{lllll} 
IFITM1 & RSAD2 & GBP4 & CMPK2 & IL15 \\
CXCL10 & CXCL11 & MX1 & OAS1 & IL7 \\
USP18 & IFIT3 & ISG15 & IFI44 & DDX60 \\
IFI44L & OASL & IFI27 & GMPR & LY6E \\
IFIT2 & ISG20 & PLSCR1 & HERC6 & LGALS3BP \\
\hline
\end{tabular}




\section{Figure Legends}

\section{Figure 1. HIV-1 infection triggers a type I IFN response in DCs.}

(A) Schematic of the human DC network project depicting derivation of monocyte-derived DCs, time series stimulation with HIV-1-GFP, LKO-GFP, LPS, and polyl:C, followed by network inference, network visualization, in silico quality control, and experimental validation.

(B) Heat map of differentially expressed genes in DCs across time (2, 8, 24 and $48 \mathrm{~h})$, selected at FDR $<0.1$ and sorted by Louvain modularity cluster (see STAR Methods).

(C) GSEA plots for the Hallmark IFN Response in DCs for all described HIV-1-GFP time points compared to mock, indicating Normalized Enrichment Score (NES) and False Discovery Rate (FDR). Enrichment is considered significant at FDR $<0.25$.

(D) Leading edge genes from GSEA for HIV-1-GFP infections at $24 \mathrm{~h}$.

See also Figure S1 and Tables S1-2. 
Figure 2

A

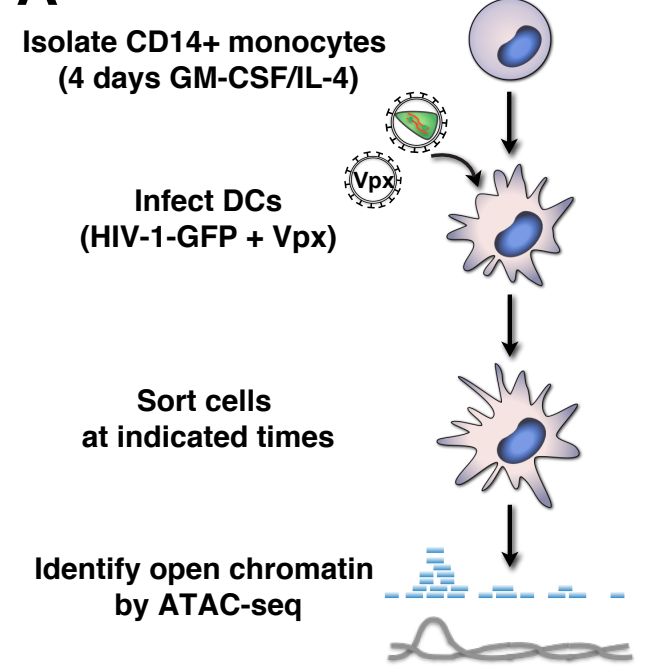

B

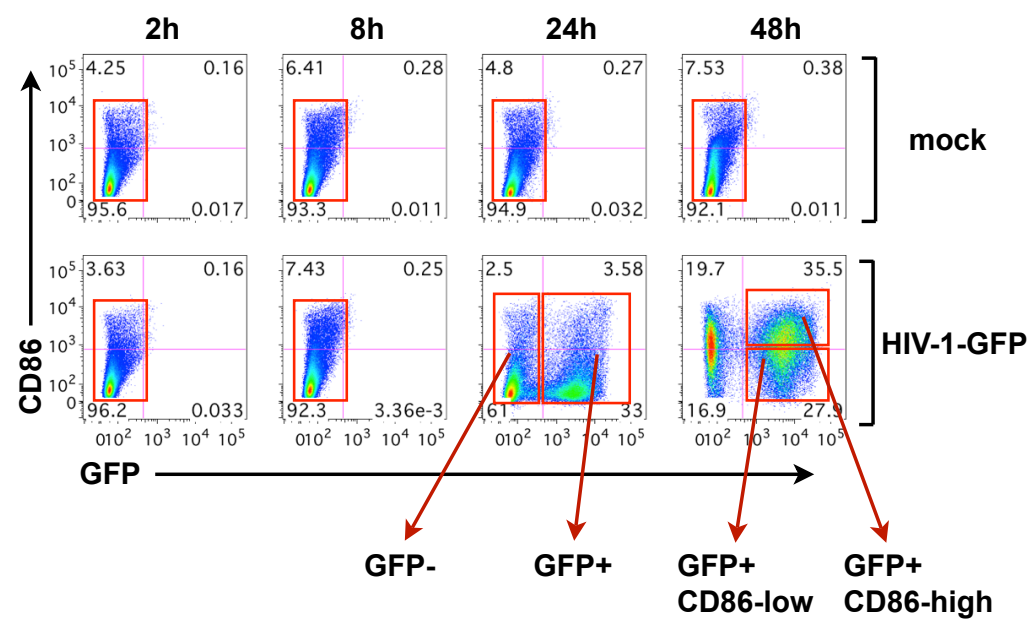

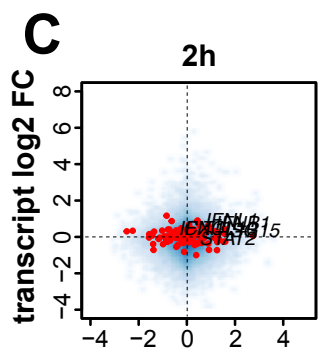
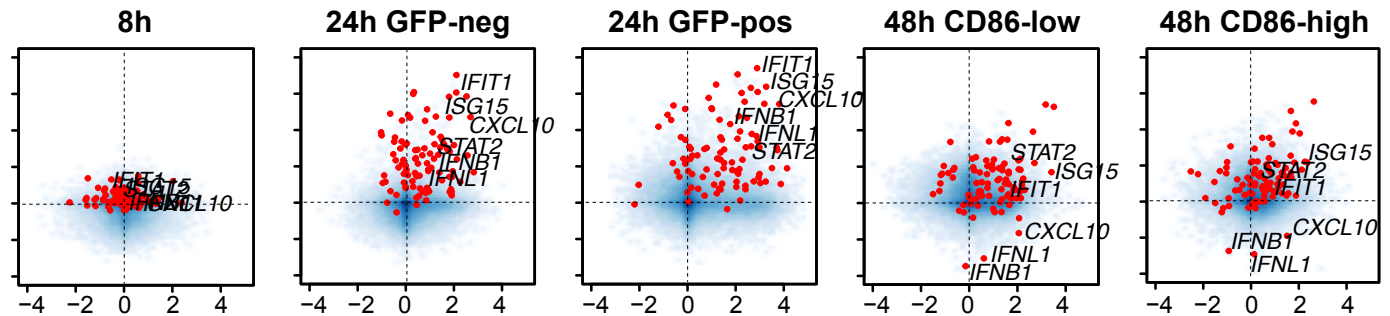

TSS peak log2 FC (ATAC-seq)

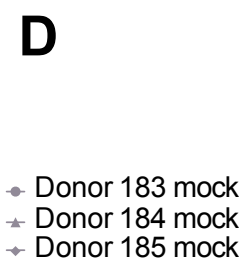

- Donor 183 HIV-1-GFP

* Donor 184 HIV-1-GFP

- Donor 185 HIV-1-GFP
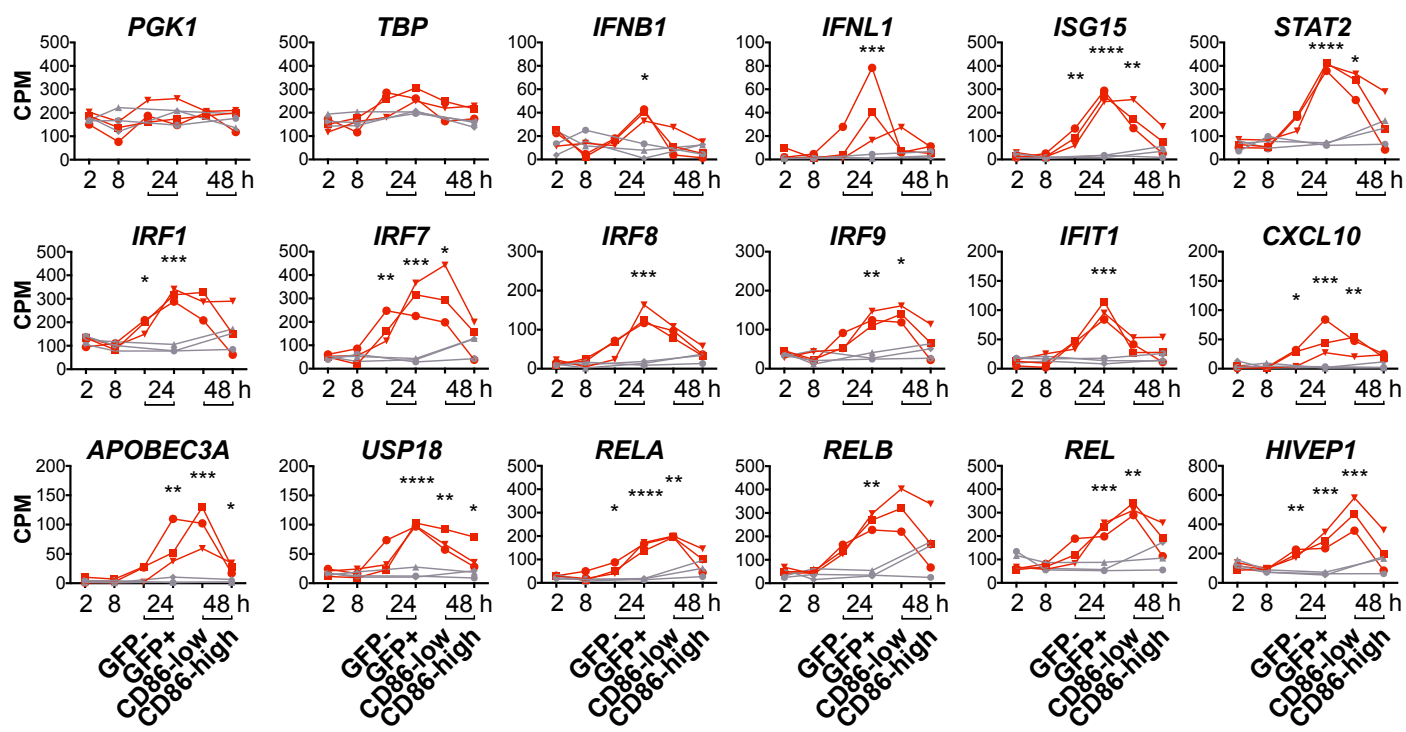
Figure 2. Transient changes in chromatin accessibility correspond to activation of the innate response during HIV infection.

(A) Illustration depicting ATAC-seq sample processing

(B) Flow cytometry plots of DCs sorted (red boxes) for ATAC-seq after infection with HIV-1-GFP

at $2,8,24$, and $48 \mathrm{~h}(\mathrm{MOI}=5)$. Plots show CD86 vs GFP expression and are representative data from 1 of 3 donors.

(C) Smooth scatter density plots of HIV-1-GFP infected DCs from sorted populations as in (B) showing the genome-wide relationship between transcript levels (RNA-seq average log2 fold change) TSS chromatin accessibility (ATAC-seq average log2 fold change). Blue: density of points across the entire genome. Red: Hallmark IFN response genes as in Figure $1 \mathrm{C}$. The position of IFNB1, IFNL1, and select ISGs are labeled for each condition.

(D) ATAC-seq gene-associated peak values shown as counts per million reads (CPM) for the indicated time points and conditions. Lines connect samples from independent donors. Statistics were calculated on log2-transformed data using a 2-way ANOVA with multiple comparisons; * $p$ $<0.05 ;{ }^{* *} p<0.01 ;{ }^{* * *} p<0.001 ;{ }^{* * * *} p<0.0001$.

See also Figure S2 and Table S3. 
Figure 3

A

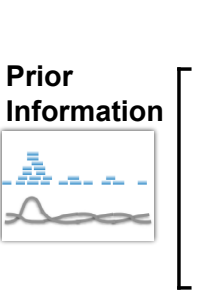

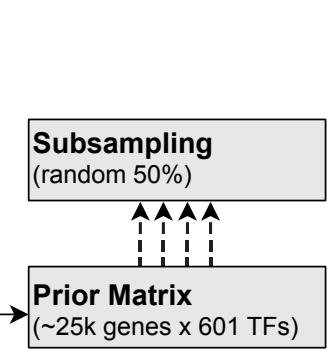

TF Accessibility
Inferelator

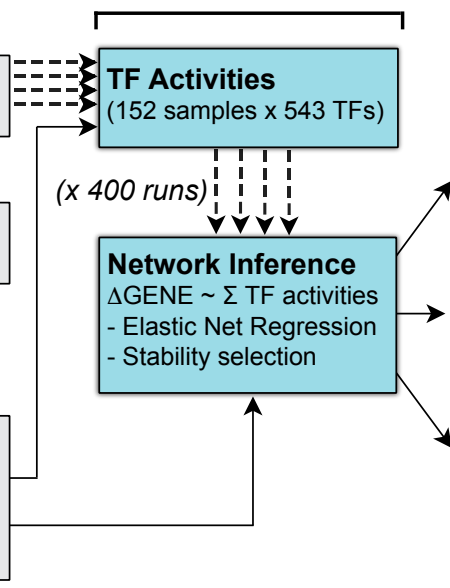

Performance Evaluation/ Quality Control

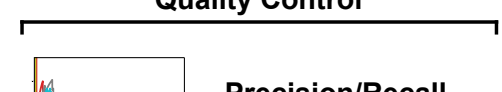

on Gold Standards:

- TRRUST

- Known ISGs

Visualization:

- Principal components

- Louvain clustering

- Hypergeometric tests

- Subnetwork mapping
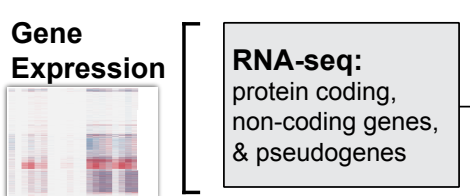

Expression Matrix

$\sim 60.5 \mathrm{k}$ target genes

x 152 samples

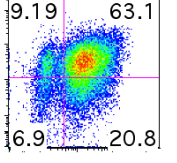

\section{Validation:}

- Knockdown

- Drug perturbation
B

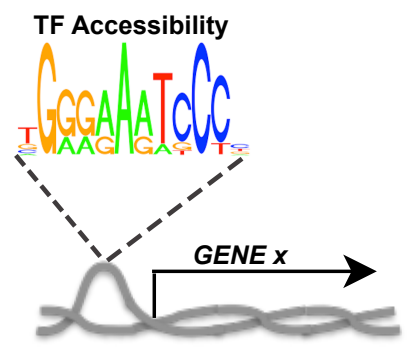

Prior:

$P(\mathrm{TF} \longrightarrow x) \quad x \approx \bar{x}$

$X=$ Gene Expression

$\mathrm{P}=$ Prior Network

A = Estimated TF Activity

$B=$ Inferred Network

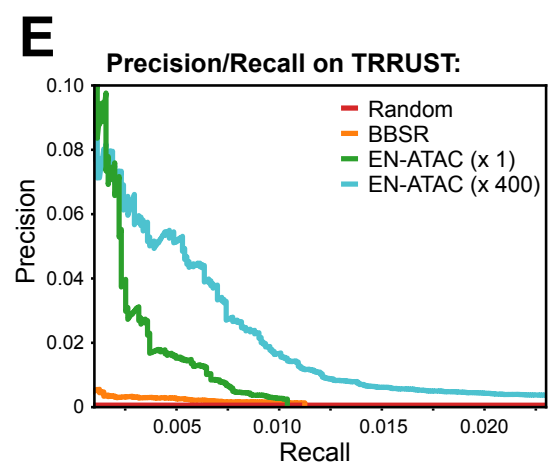

C
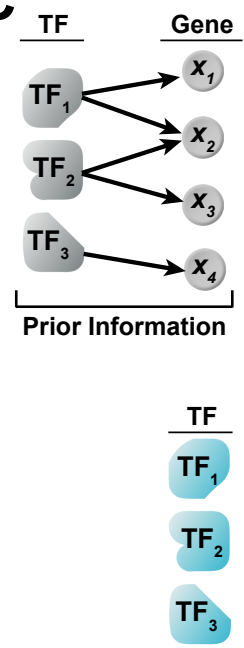

Estimated TF Activity:

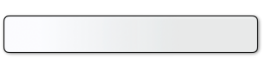

$\mathrm{TF}_{3}$
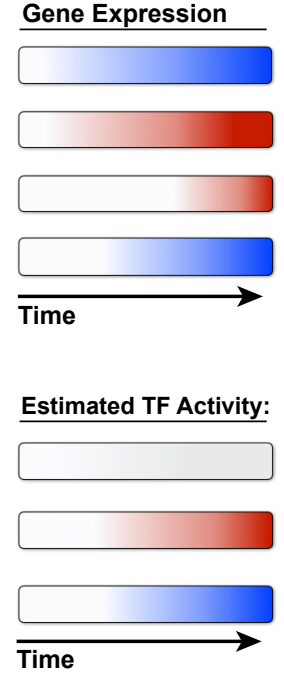

D

F

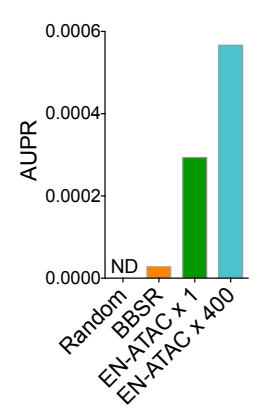

G

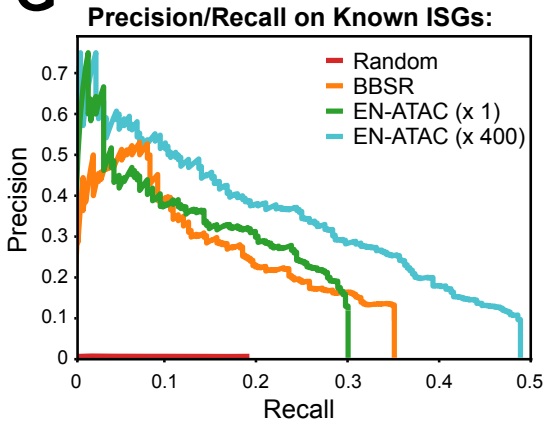

H

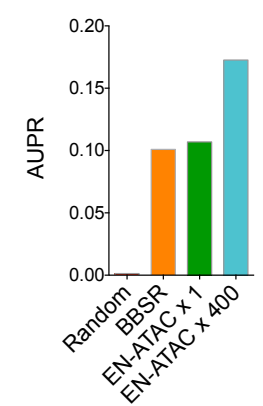


Figure 3. Repeated subsampling of prior information improves network inference.

(A) Pipeline for network inference. Prior information (TF accessibility) and gene expression time series data were used as inputs for the Inferelator. Output networks underwent performance evaluation and quality control as indicated.

(B) The prior matrix denotes a possible connection between a TF and gene $X$ if that TF's binding motif is found in accessible chromatin upstream $(-1 \mathrm{~kb})$ or within gene $\mathrm{X}$.

(C) Schematic illustrating how TF activity is estimated by summing predicted target expression over time $($ red = increase; blue = decrease $)$.

(D) TF and gene target connections in the prior network are pruned using an Elastic Net regression to yield the final inferred network.

(E) Precision/Recall plots and area under precision/recall (AUPR) curves (F) for the indicated networks scored against the TRRUST database (see STAR Methods).

(G) Precision/Recall plots and AUPR curves $(\mathrm{H})$ for the indicated networks scored against known ISGs (Schoggins et al., 2011).

See also Figure S3 and Table S4. 
Figure 4

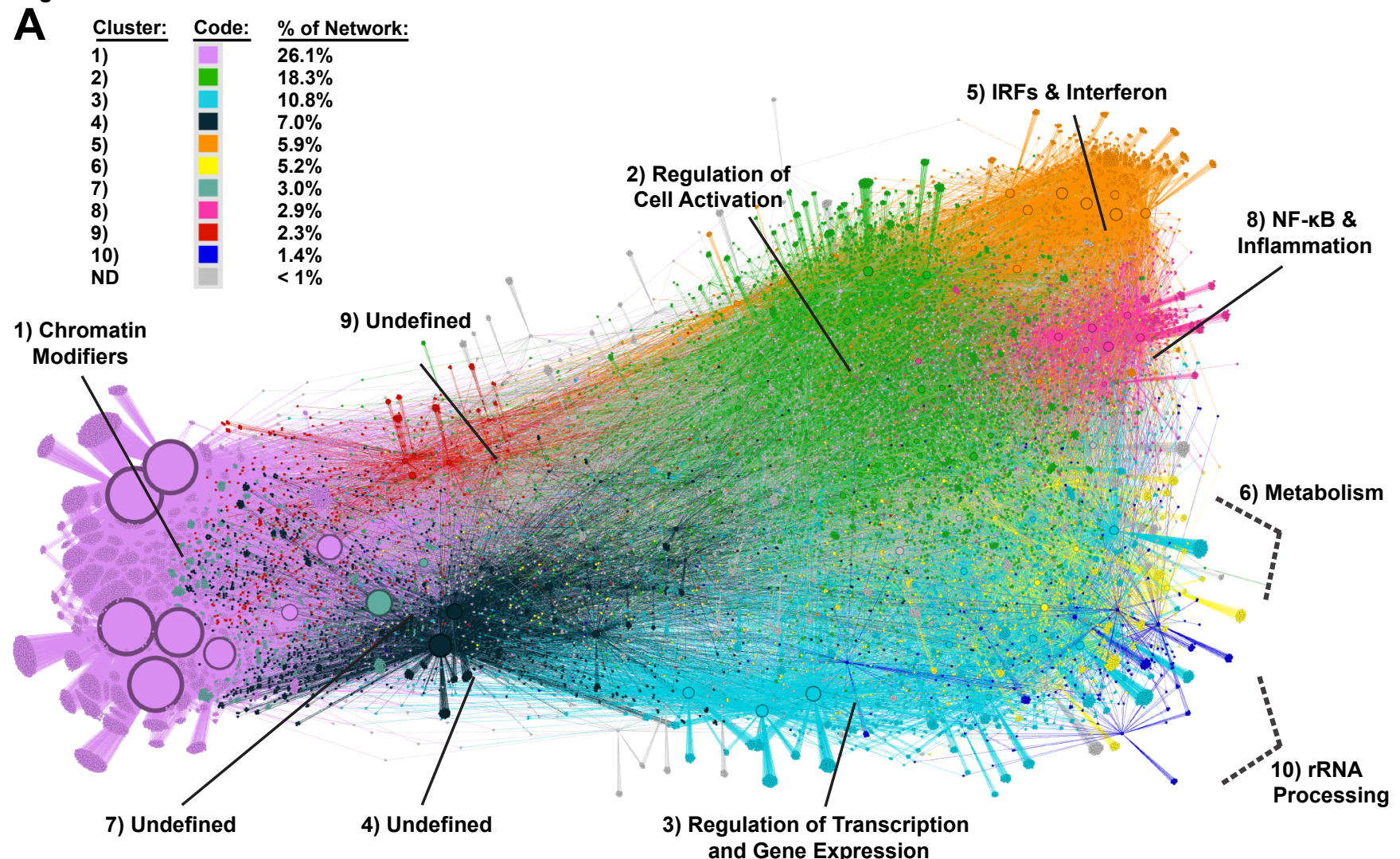

B

Reactome Pathway Analysis
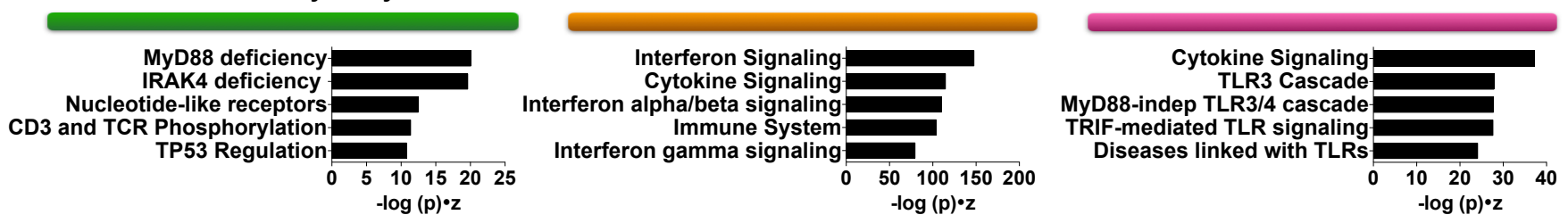

\section{Genome Browser PWMs}

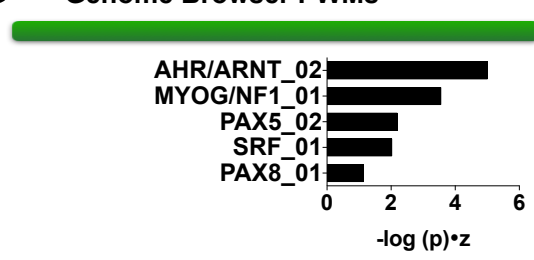

\section{TF Perturbations}
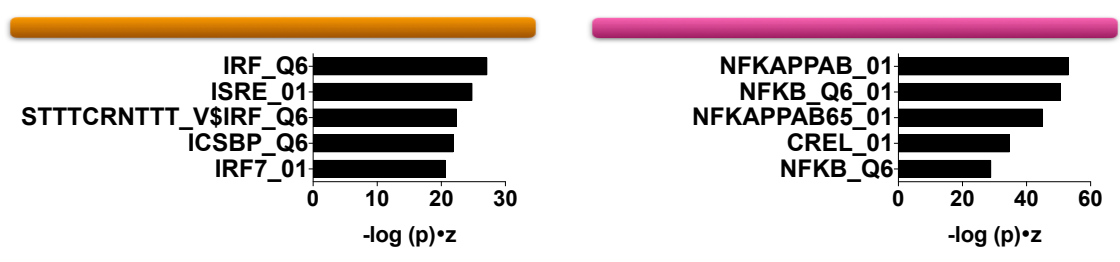

\begin{tabular}{|c|c|}
\hline $\begin{array}{l}\text { RARA ACTIVATION (human)- } \\
\text { E2F8 KO (mouse) } \\
\text { MAF siRNA (human) } \\
\text { GATA6 KO (mouse) } \\
\text { MAF OE (human) }\end{array}$ & 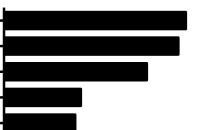 \\
\hline & \begin{tabular}{|llll}
0 & 20 & 40 & 60 \\
& $-\log (p) \cdot z$ &
\end{tabular} \\
\hline
\end{tabular}

\section{E JASPAR \& TRANSFAC PWMs}

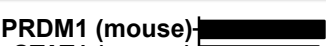

STAT1 (mouse) HIIIIIIIIIIII

FOXM1 (human)

MIR210 (human)

NFAT2 (human)

\begin{tabular}{llll}
\hline & 5 & 10 & 15
\end{tabular}

RELA (human)- "IIIIIIIIIIIIII

RELB (human)

SND1 (human)

NFKB1 (human) - m!1!1!

HNF1B (mouse)

\begin{tabular}{llll}
\hline 0 & 10 & 20 & 30
\end{tabular}


Figure 4. Modularity clustering of the network defines TF and gene target clusters predicted to control distinct biological functions.

(A) EN-ATAC network topology in DCs visualized through Gephi software. Clusters were named according to pathway and motif enrichment (see STAR Methods). Arrows indicate transcriptional regulatory events between TFs and targets and do not specify positive or negative effects on target expression. Node size denotes relative number of edges. Clusters are color-coded based on Louvain clustering and ranked based on their decreasing size ( from 1 to 10) (based on \% of genes in each cluster vs total number of genes)

(B-D) Enrichment of pathways from Reactome (B), Genome Browser TF position weight matrices (PWM) (C), TF Perturbations (D), and JASPAR and TRANSFAC PWMs (E) for Clusters 2, 5, and 8 (color-coded as in panel A). Ranking is by Enrichr combined score: (-log (p) ${ }^{*}$ z-score).

See also Figure S4 and Tables S5-6. 

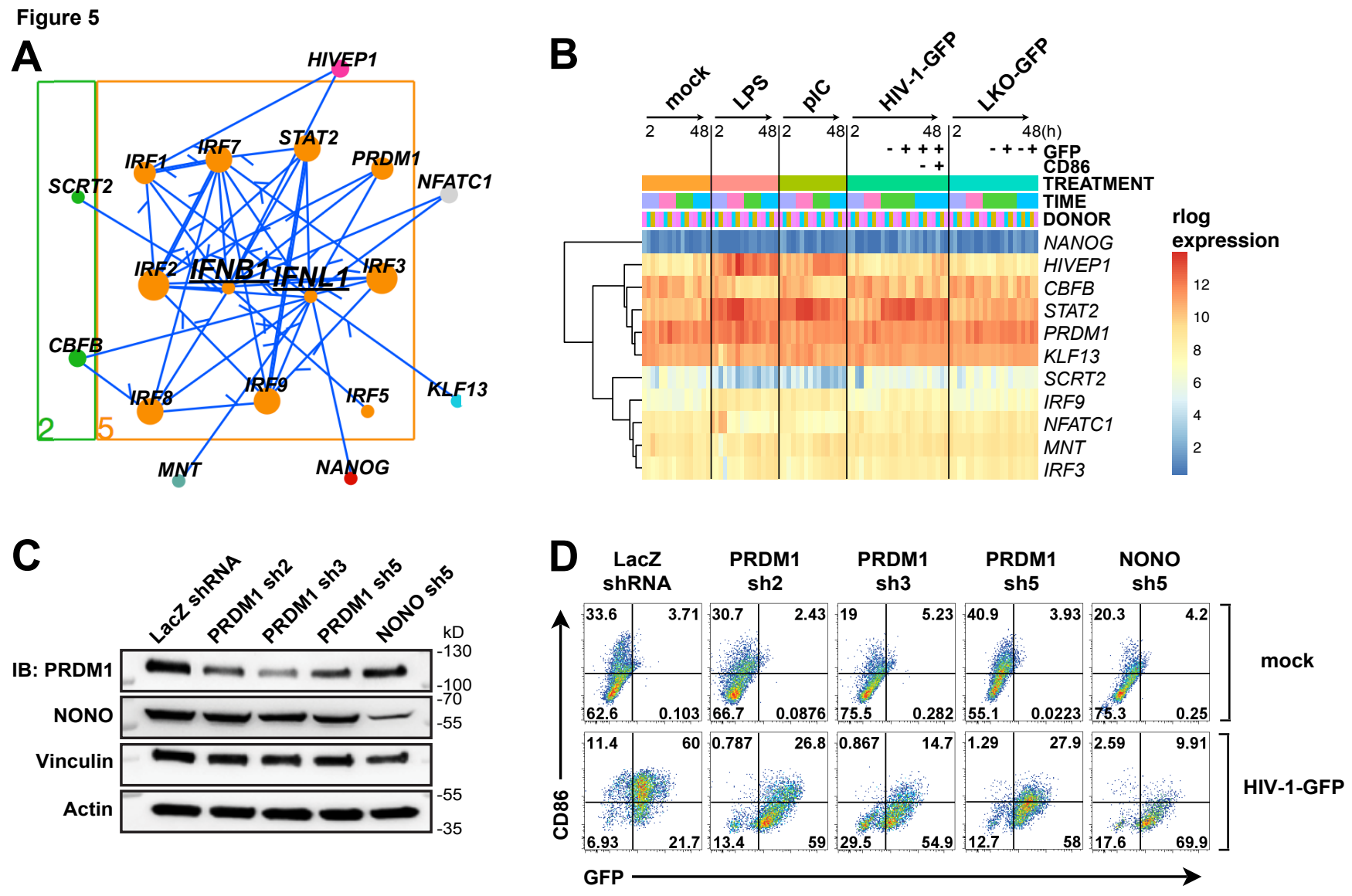
E $\rightarrow$ control sh (LacZ)
$\rightarrow$ NONO $\operatorname{sh} 5$
$₫$ PRDM1 sh2
$\rightarrow$ PRDM1 sh3
$\rightarrow$ PRDM1 sh5

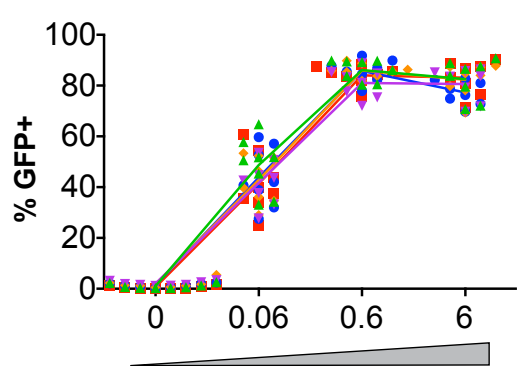

HIV-1-GFP
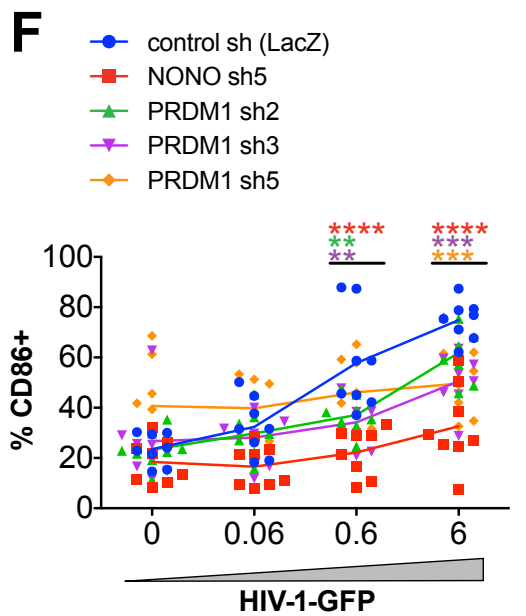

G

$$
\begin{aligned}
& \circ \text { control sh (LacZ) } \\
& \square \text { NONO sh5 } \\
& \triangle \text { PRDM1 sh2 } \\
& \nabla \text { PRDM1 sh3 } \\
& \diamond \text { PRDM1 sh5 }
\end{aligned}
$$

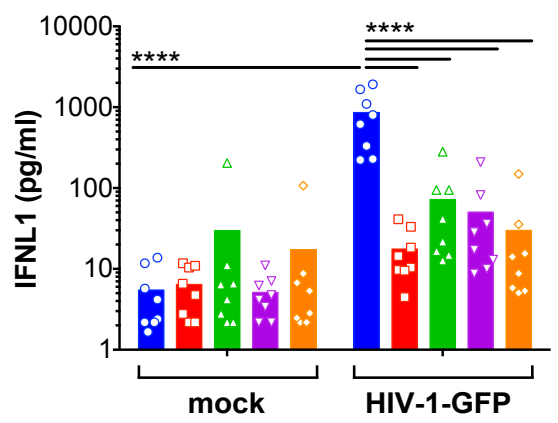


Figure 5. Subnetwork exploration uncovers positive and negative regulators of IFN and ISGs.

(A) Jupyter widget subnetwork view (see STAR Methods) of predicted upstream regulators of IFNB1 and IFNL1. Arrows indicate transcriptional regulatory events between TFs and targets and do not specify positive or negative effects on target expression. Node size denotes relative number of edges. Nodes are color coded by network cluster (see Figure 4).

(B) Heat map of gene expression for the indicated TFs during treatment with LPS, pIC, HIV-1GFP, and LKO-GFP at 2, 8, 24, and 48 h. (C) Immunoblots of DC lysates after treatment with the indicated shRNAs.

(D) Flow cytometry of CD86 vs GFP expression in shRNA-modified DCs $48 \mathrm{~h}$ after mock treatment or infection with HIV-1-GFP.

$(E-F)$ Plots showing \%GFP+ $(E)$ and \%CD86+ $(F)$ from DCs treated as in (D).

(G) ELISA of IFNL1 expression in supernatants from shRNA-modified DCs that were mock treated or infected with HIV-1-GFP for 48 h. For E-G, plots represent pooled data from 8 donors. ${ }^{* *} p<0.01 ;{ }^{* * *} p<0.001 ;{ }^{* * *} p<0.0001$

See also Figure S5. 
Figure 6

A

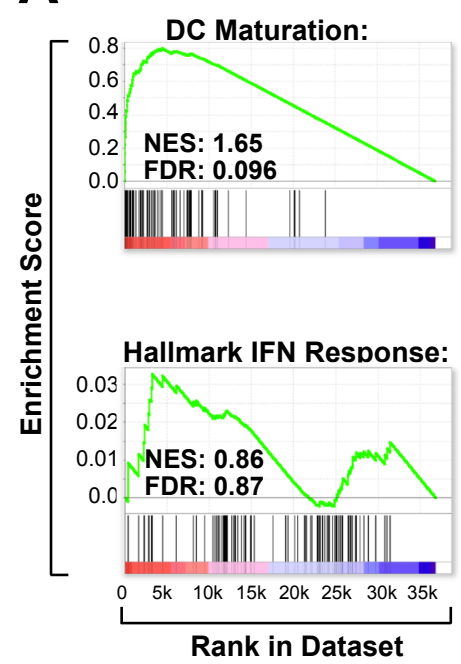

D

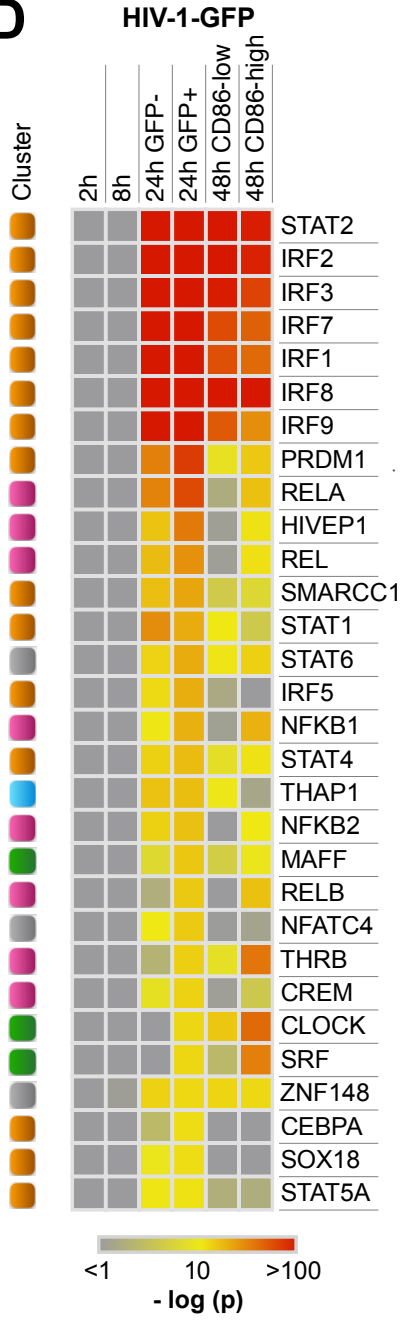

B
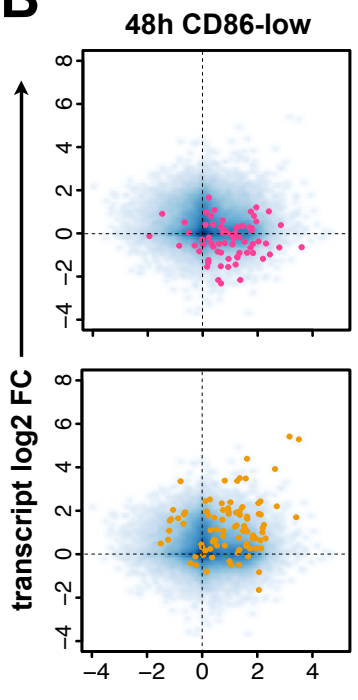

TSS peak log2 FC (ATAC-seq)
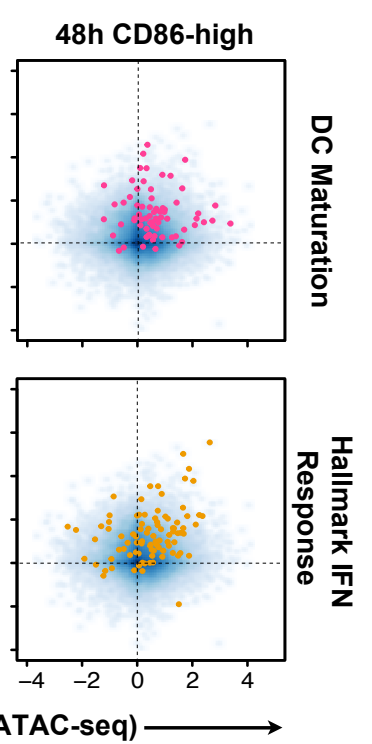

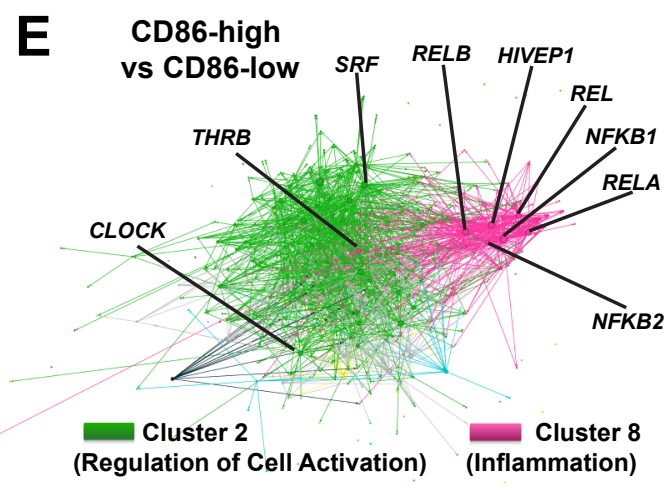

G Protein-Protein Interactions:

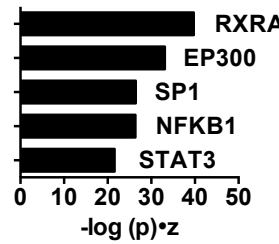

I Single Gene Perturbations:

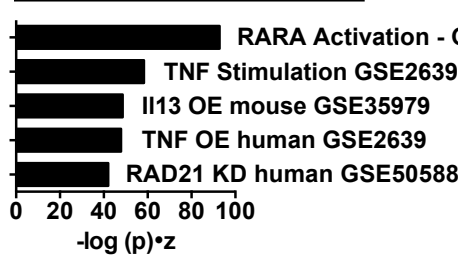

C
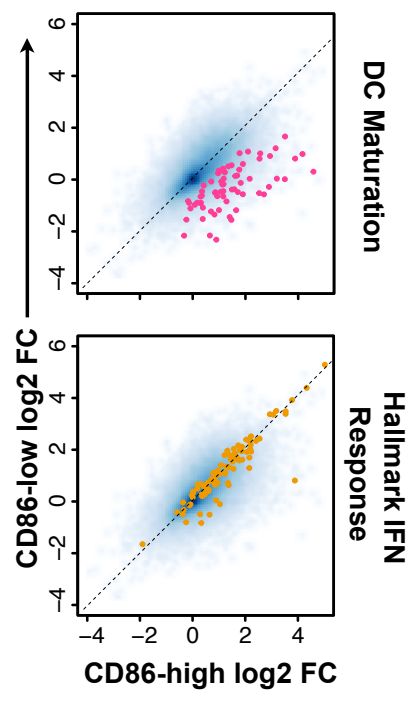

F

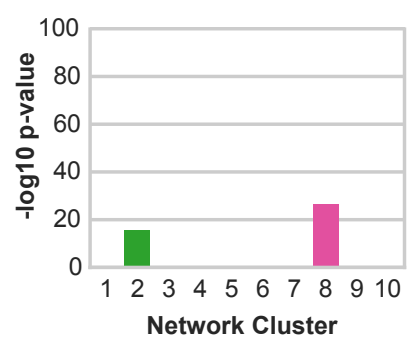

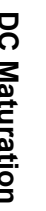

Reactome:

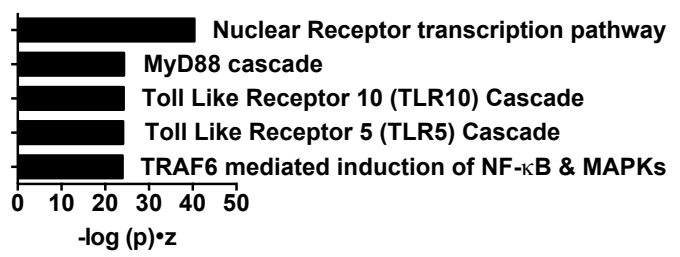

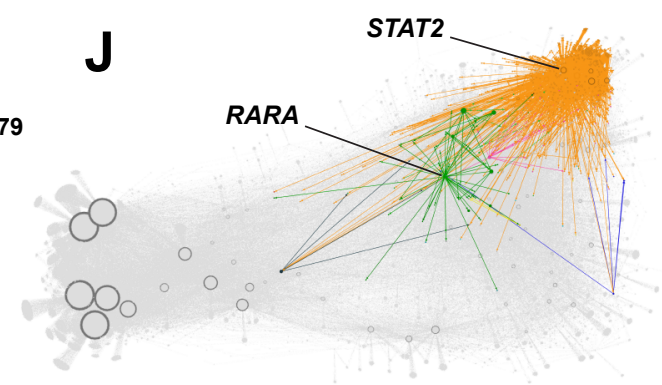


Figure 6. Mature DCs cannot be identified based on an IFN signature alone.

(A) GSEA plots comparing gene expression data for CD86-high (mature) vs CD86-low (immature) DCs. Enrichment is considered significant at FDR $<0.25$

(B) Smooth scatter density plots of transcript levels (RNA-seq) vs. TSS chromatin accessibility (ATAC-seq) in HIV-1-GFP infected DCs for CD86-low and CD86-high populations. Blue density marks the entire genome. Genes in the DC Maturation gene set (pink) and the Hallmark IFN Response gene set (orange) are highlighted in the top and bottom plots, respectively.

(C) Log2 normalized gene expression plotted for the CD86-low population (y-axis) and CD86high population sorted at 48 h. DC Maturation genes (pink) and Hallmark IFN Response genes (orange) are shown as in (B).

(D) Heat map of hypergeometric scores for TF enrichment displaying HIV-1-GFP compared to mock at 2 h, 8 h, 24 h (GFP-), 24 h (GFP+), 48 h (CD86-low), and 48 h (CD86-high). TFs were ranked in descending order according to the $24 \mathrm{~h}$ GFP+ condition. Heat map is color coded according to -log p-value of TF enrichment.

(E) Differential gene expression contrast between CD86-high and CD86-low populations as visualized by Gephi software, highlighting edges in Clusters 2 and 8 . Ranking is by Enrichr combined score: $\left(-\log (p){ }^{*} z\right.$-score $)$.

(F) Enrichment of differentially expressed genes separated by network cluster for the CD86-high vs CD86-low contrast as in (E).

Potential Protein-Protein interactions (G), Reactome pathway enrichment $(H)$, and Single Gene Perturbations (I) predicted from differentially expressed genes in the CD86-high vs CD86-low contrast.

(J) Network connections for RARA and STAT2 visualized by Gephi software, color coded by contrast, and superimposed onto a grey network backdrop.

See also Figure S6. 
Figure 7
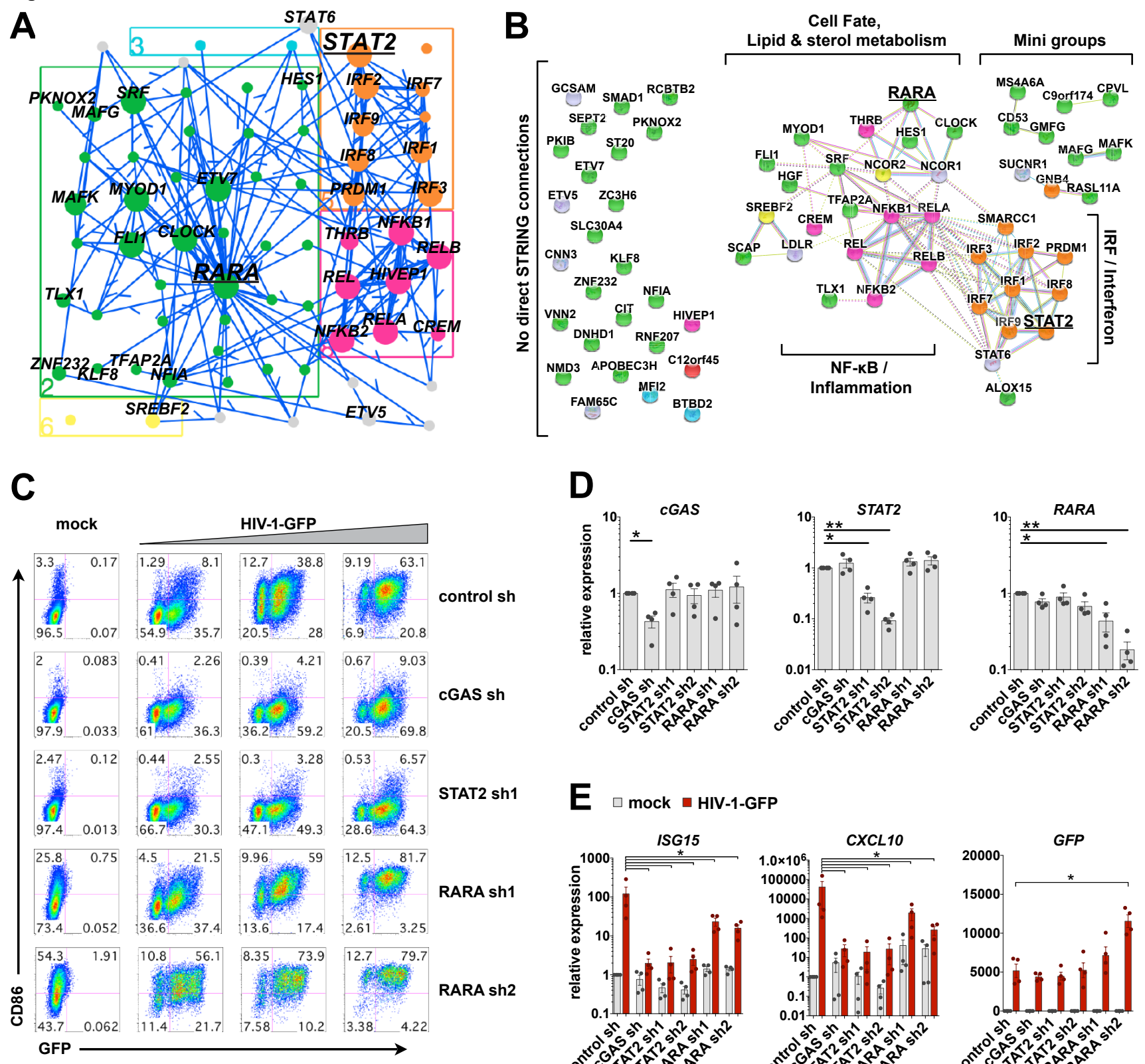

E $\square$ mock $\square$ HIV-1-GFP
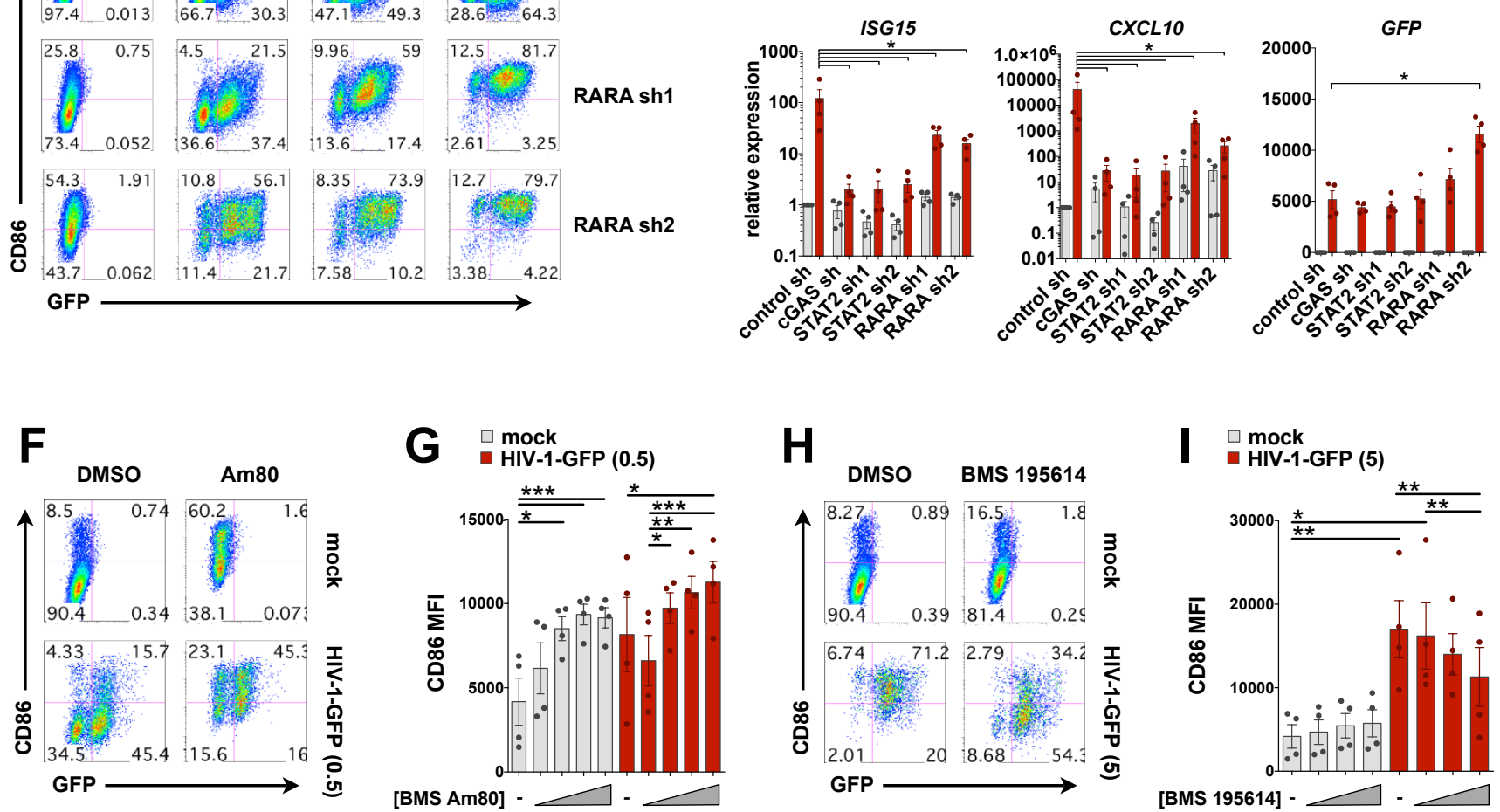

[BMS 195614] - 


\section{Figure 7. RARA is a negative regulator of DC maturation.}

(A) Jupyter widget subnetwork view (see STAR Methods) showing edges between RARA, its predicted targets, STAT2, and the remaining top 30 TFs ranked by hypergeometric p-value. Arrows point from TFs to targets but do not suggest positive or negative activity.

(B) STRING database connections for nodes shown in (A) including NCOR1 and NCOR2. Brackets indicate groups and biological pathways. Nodes connected in the EN-ATAC network with no direct STRING connections are shown to the left. In (A) and (B) nodes are color coded by network cluster.

(C) Flow cytometry plots of DCs modified by shRNA that were mock infected or challenged with HIV-GFP at day 4 for $48 \mathrm{~h}$. Plots show CD86 vs GFP expression and represent 1 of 4 donors $(\mathrm{MOI}=0.5,1.5,5)$

(D) qPCR validation of target knockdown in DCs modified by the indicated shRNAs.

(E) qPCR of ISG15, CXCL10, and GFP expression in shRNA-modified DCs that were either mock treated or infected with HIV-1-GFP $(\mathrm{MOI}=5)$ for $24 \mathrm{~h}$.

(F) Flow cytometry of DCs treated with vehicle or the RARA agonist Am80 $(10 \mu \mathrm{M}) \pm$ infection with HIV-1-GFP for $48 \mathrm{~h}$.

(G) Pooled data of CD86 MFI in DCs from 4 donors treated as in (F). Am80 concentrations = 10nM, 100nM, $1 \mu \mathrm{M}, 10 \mu \mathrm{M}$.

(H) Flow cytometry of DCs treated with vehicle or the RARA antagonist BMS $195614(10 \mu M) \pm$ infection with HIV-1-GFP for $48 \mathrm{~h}$.

(I) Pooled data of CD86 MFI in DCs from 4 donors treated as in (H). BMS 195614 concentrations $=1 \mathrm{nM}, 100 \mathrm{nM}, 10 \mu \mathrm{M}$. For $\mathrm{D}, \mathrm{E}, \mathrm{G}$, and I: $\mathrm{n}=4$ donors. ${ }^{*} \mathrm{p}<0.05 ;{ }^{* *} \mathrm{p}<0.01$; ${ }^{* * *} p<0.001$. Data represent mean \pm SEM.

See also Figure S7. 


\section{STAR Methods}

\section{CONTACT FOR REAGENT AND RESOURCE SHARING}

Further information and requests for resources and reagents should be directed to and will be

fulfilled by the Lead Contact, Mickaël M. Ménager (mickael.menager@institutimagine.org).

\section{EXPERIMENTAL MODEL AND SUBJECT DETAILS}

\section{Cell lines and blood-derived dendritic cells}

To generate monocyte-derived immature DCs, we acquired leukocytes from de-identified normal human donors from a variety of sources (Bloodworks Northwest, Renton, WA, USA; New York Blood Center, New York, NY, USA; ARUP Blood Services, Sandy, UT, USA; and from venipunctures (approved by the Institut National de la Santé et de la Recherche Médicale ethics committee, Paris, France). The authors cannot report on the sex, gender, or age of the donors since the samples were de-identified and donors remain anonymous. Peripheral blood mononuclear cells (PBMCs) were layered over Ficoll-Paque Plus (GE Healthcare). CD14+ monocytes from PBMC buffy coats were isolated with anti-human CD14 magnetic beads (Miltenyi) and cultured in RPMI (Thermo Fisher) containing 10\% heat-inactivated fetal bovine serum (FBS, Peak Serum, Inc), $50 \mathrm{U} / \mathrm{ml}$ penicillin, $50 \mu \mathrm{g} / \mathrm{ml}$ streptomycin (P/S, Thermo Fisher), 10 mM HEPES (Sigma), 2-Mercaptoethanol (Thermo Fisher), and 2 mM L-glutamine (Thermo Fisher), in the presence of recombinant human GM-CSF at $10 \mathrm{ng} / \mathrm{ml}$ and IL-4 at $50 \mathrm{ng} / \mathrm{ml}$ (Peprotech). Multiple lots of FBS were tested to identify batches leading to minimal baseline induction of CD86 over the course of DC differentiation. Fresh media and cytokines were added to cells ( $40 \%$ by volume) one day after CD14+ cell isolation. On day 4 , cells were collected, resuspended in fresh media with cytokines used for infection or stimulation. Immature DCs on day 6 were routinely assessed by flow cytometry surface marker staining to be CD11c+ 
(Thermo Fisher Cat\# 17-0116-42, RRID:AB_1659668), HLA-DR+ (BioLegend Cat\# 307607, RRID:AB_314685), DC-SIGN+ (R and D Systems Cat\# FAB161P,RRID:AB_357064), and CD86- (eBioscience Cat\# 15-0869-42 RRID:AB_11042003). DC experiments were performed using biological replicates from blood-derived cells from multiple individual donors as indicated in the figure legends.

293FT female cells (Life Technologies Cat\# R70007, RRID:CVCL_6911) were cultured in Dulbecco's modified Eagle's medium (DMEM, Thermo Fisher) that was supplemented with 10\% FBS, P/S, 10mM HEPES, and supplemented with $0.1 \mathrm{mM}$ MEM non-essential amino acids (Thermo Fisher), $6 \mathrm{mM}$ glutamine, and $1 \mathrm{mM}$ sodium pyruvate (Thermo Fisher).

HL116 male cells were cultured in DMEM supplemented with 10\% FBS, P/S, 10mM HEPES, $0.1 \mathrm{mM}$ MEM non-essential amino acids, $6 \mathrm{mM}$ glutamine, $1 \mathrm{mM}$ sodium pyruvate, and HAT supplement (Thermo Fisher; hypoxanthine $(5 \mathrm{mM})$, aminopterin $(20 \mu \mathrm{M})$ and thymidine $(0.8$ $\mathrm{mM})$ ) diluted 1:50 as recommended.

All cell lines were thawed from early passages, kept in culture no longer than 4 weeks, and were regularly tested for mycoplasma contamination (every 6 months). All cells were maintained at $37^{\circ} \mathrm{C}$ and $5 \% \mathrm{CO} 2$.

\section{METHOD DETAILS}

\section{Plasmids}

HIV-1-GFP has been used previously to study immune responses in human DCs (Manel et al., 2010) and is env- vpu- vpr- vif- nef-, with the GFP open reading frame in place of nef. We generated virus like particles packaging Vpx from the plasmid pSIV3+ (based on SIVmac251, GenBank acc. no. M19499), which has been described elsewhere (Mangeot et al., 2000). 
Target sequences for shRNA vectors are listed in Table S7. All lentiviral constructs were transformed into Stbl3 bacteria (ThermoFisher) for propagation of plasmid DNA. All plasmids were prepped and purified using Nucleobond Xtra Maxi Kit (Takara) or similar maxi prep reagents.

\section{Virus and virus-like particle production}

To produce recombinant lentiviral vectors, reporter viruses, and Vpx-containing virus-like particles, we transfected plasmids into 293FT cells as previously described (Johnson et al., 2018). Briefly, the day before transfection, cells were seeded onto poly-L-lysine (MP Biomedicals) -coated $15 \mathrm{~cm}$ plates to be $70-80 \%$ at the time of transfection. Cells were transfected with a total of $22.5 \mu \mathrm{g}$ DNA using PEImax (Polysciences, Inc.) at a ratio of 1:2 (DNA:PEI). For lentiviral vectors, plasmid amounts were 3.4 $\mu \mathrm{g}$ CMV-VSV-G (Addgene plasmid \#8454), $9 \mu \mathrm{g}$ psPax2 (Addgene plasmid \#12260), and 10.1 $\mathrm{gg}$ transgene (LKO.1 controls or shRNA construct). For HIV reporter viruses plasmid amounts were $3.4 \mu \mathrm{g}$ CMV-VSV-G and 19.1 $\mu \mathrm{g}$ HIV cassette. Virus-like particles containing Vpx were produced using 3.4 $\mu \mathrm{g}$ CMV-VSV-G and 19.1 $\mathrm{\mu g}$ pSIV3+ (Mangeot et al., 2000). The morning after transfection, cells were washed once and fed with $30 \mathrm{ml}$ of fresh media. Supernatants were harvested 32-36 $\mathrm{h}$ after feeding 293FTs and then passed through $0.45 \mu \mathrm{m}$ syringe filters (Corning) to remove debris.

Supernatants of reporter virus and lentiviral vectors were either used fresh for transduction or concentrated by ultracentrifugation by spinning in conical tubes (Beckman) at $25 \mathrm{~K} \mathrm{rpm}$ for $2 \mathrm{~h}$ at $4{ }^{\circ} \mathrm{C}$ in an SW32 swing-bucket rotor (Beckman). Lentiviral pellets were thoroughly resuspended ( $\sim 30$-fold concentrated) in DC media without cytokines. Before transducing cells, insoluble material was clarified from lentivirus stocks by centrifuging at 300 rcf for 3 min at $4 \mathrm{C}$. Viral stocks were frozen at $-80^{\circ} \mathrm{C}$ and used as indicated below. 


\section{Perturbation of DCs}

To genetically modify DCs, we isolated CD14+ monocytes from buffy coats as described above and transduced them with lentiviral vectors in the presence of virus-like particles packaging Vpx similar to previously described protocols (Johnson et al., 2018; Manel et al., 2010). Briefly, monocytes were resuspended in complete DC media containing GM-CSF (10 ng/ml), IL-4 (50 $\mathrm{ng} / \mathrm{ml}$ ), polybrene (Sigma, $1 \mu \mathrm{g} / \mathrm{ml}$ ), and supernatants containing Vpx particles $(1 \mathrm{ml}$ supernatant for $1 \times 10^{\wedge} 7$ cells) and then were plated in either $10 \mathrm{~cm}$ dishes $\left(6 \times 10^{\wedge} 6\right.$ cells in $9 \mathrm{ml}$ media) or 96 well plates $(160,000$ cells in $150 \mu \mathrm{l}$ media). Roughly 30 min after plating, clarified concentrated lentiviral stocks were added to transduce cells. We routinely observed that 95$99 \%$ of cells could be transduced using $150 \mu$ concentrated vector in $10 \mathrm{~cm}$ plates or $5 \mu \mathrm{l}$ concentrated vector per well in 96 well plates.

\section{Infections and stimulations}

DCs were infected or stimulated with innate agonists between day 4 and day 6 after differentiation. DCs were counted on day 4 and resuspended at 800,000 cell per $\mathrm{ml}$ in fresh medium with GM-CSF, IL-4, and polybrene $(1 \mu \mathrm{g} / \mathrm{ml})$ and then reseeded into appropriate culture vessels. For most assays, DCs were infected at a density of 800,000 cells per ml by diluting virus in DC media (without cytokines or polybrene) to a final volume normalized to controls. Innate and inflammatory stimuli were used at the indicated concentrations: polyl:C (InvivoGen, $10 \mu \mathrm{g} / \mathrm{ml}$ ); LPS (List Biological Laboratories, INC, $1 \mathrm{ng} / \mathrm{ml}$ ).

\section{Flow cytometry}

Infected or stimulated DCs were washed with phosphate buffered saline (PBS, Corning) and then exposed to LIVE/DEAD violet (ThermoFisher) in PBS for $15 \mathrm{~min}$ at $4{ }^{\circ} \mathrm{C}$ in the dark. Cells were either simultaneously stained for surface markers or first washed with PBS and then stained in FACS buffer containing 1\% Bovine Serum Albumin (BSA, Roche) and 1 mM EDTA in 
PBS for $15-30$ min in the dark at $4{ }^{\circ} \mathrm{C}$. Cells were then washed with PBS and fixed with $0.5 \%$ paraformaldehyde (Electron Microscopy Sciences) diluted in PBS. Cells were either sorted on a FACSAria II or data was acquired on an LSR II flow cytometer (BD Biosciences). Data were then analyzed using FlowJo software (FlowJo LLC).

\section{RNA-seq}

DCs from multiple donors were infected or stimulated as indicated in the figure legends, stained with anti-human CD86 and a live-dead viability dye and then sorted into serum on a FACSAria II (BD Biosciences). Cells were lysed in TRIzol reagent (Thermo Fisher), RNA was isolated according the manufacturer's instructions, and then samples were submitted to HudsonAlpha Institute for Biotechnology (https://hudsonalpha.org/) for library preparation and RNAsequencing.

50bp-length single-end sequences were aligned to the human genome (hg38) using STAR version 2.4.2a. Samtools 0.1.19 was used to filter alignments to a MAPQ score threshold of 30 . Counts per gene were called using featureCounts version 1.4.6 and gencode v24 genome annotation. Samtools 0.1.19 was used to filter alignments to a MAPQ score threshold of 30 . We confirmed the presence of HIV-1 or HIV-2 sequences in infected samples yet these sequences were not taken into account during differential expression analysis. Differential expression analysis was performed separately for each RNA-seq experiment using DESeq2 version 1.10.1 and $\mathrm{R}$ version 3.2.3, using time and treatment together as the design parameter. Genes were considered differentially expressed in a given comparison if the FDR-adjusted p-value was below 0.1 . 


\section{ATAC-seq}

DCs from three unique donors were infected in the presence of $\mathrm{Vpx}$ to match infection conditions as performed for RNA-seq at 2, 8, 24, and $48 \mathrm{~h}$ after infection. Cells were stained for LIVE/DEAD violet and CD86 to assess activation status and sorted on a FACSAria II (BD Biosciences) according to the gating strategy established for RNA-seq. 50,000 sorted DCs from each condition were immediately prepped for ATAC-seq (Buenrostro et al., 2013). Cells were pelleted by spinning at $500 \mathrm{~g}$ for $5 \mathrm{~min}$ at $4{ }^{\circ} \mathrm{C}$, resuspended in cold PBS, and then centrifuged again at $500 \mathrm{~g}$ for $5 \mathrm{~min}$ at $4{ }^{\circ} \mathrm{C}$. Cell pellets were lysed in cold lysis buffer $(10 \mathrm{mM}$ Tris- $\mathrm{HCl}, \mathrm{pH}$ 7.4, $10 \mathrm{mM} \mathrm{NaCl}, 3 \mathrm{mM} \mathrm{MgCl} 2$ and 0.1\% IGEPAL CA-630) and immediately spun at $500 \mathrm{~g}$ for 10 min at $4{ }^{\circ} \mathrm{C}$. The nuclear pellet was used directly for the transposition reaction by resuspending in a reaction mix $(20 \mu \mathrm{L} 2 \times$ TD buffer, $2 \mu \mathrm{L}$ Tn5 transposase (Nextera DNA Library Prep Kit, Illumina) and $18 \mu \mathrm{L}$ nuclease-free water). Transposition was performed at 37 ${ }^{\circ} \mathrm{C}$ for $30 \mathrm{~min}$ and then samples were immediately processed using MinElute kit (Qiagen) to isolate DNA.

To generate ATAC-seq libraries for sequencing, DNA fragments from MinElute samples were amplified by PCR using NEBNext PCR master mix (New England Biolabs) and custom Nextera primers (Buenrostro et al., 2013) (and Table S7) using the following conditions: $72{ }^{\circ} \mathrm{C}$ for $5 \mathrm{~min}$; $98{ }^{\circ} \mathrm{C}$ for $30 \mathrm{~s}$; and then cycling at $98^{\circ} \mathrm{C}$ for $10 \mathrm{~s}, 63^{\circ} \mathrm{C}$ for $30 \mathrm{~s}$ and $72{ }^{\circ} \mathrm{C}$ for $1 \mathrm{~min}$. To stop amplification before saturation in order to reduce bias, we monitored the PCR reaction using SYBR Green (Thermo Fisher) during qPCR. Samples were cycled a total of 12-15 times.

Libraries were sequenced on a NextSeq 500 (Illumina) using a 150 cycle high output kit. Unique sequence read pairs were aligned to the human genome (hg38) using bowtie2 (2.2.3), filtered based on mapping score (MAPQ > 30, Samtools (0.1.19)), and duplicates removed (Picard version 1.120). Only pairs that aligned uniquely and concordantly to non-mitochondrial human chromosomes were retained. 
For establishing the network prior, a merged ATAC-seq Sam file was generated by combining all ATAC-seq conditions. Peak calling was performed with Peakdeck version 1.1 (parameters bin 160, -STEP 25, -back 5000, -npBack 10000) using the start and end locations of the pairs to define fragment lengths, with a p-value of $1 e-4$ as the cutoff, outputting 87,681 unique, nonoverlapping, high-confidence peaks. Putative binding events were discovered by finding motif occurrences using HOCOMOCO version 10 (Kulakovskiy et al., 2013) as the motif database, with position weight matrices for 601 of 640 human TFs discovered using FIMO (Memesuite version 4.10.1) with a p-value cutoff of $1 \mathrm{e}-4$. When scored against our gold standards (see below) HOCOMOCO performed better than the curated CisBP 2.0 motif database (Weirauch et al., 2014) composed of JASPAR (Mathelier et al., 2016), TRANSFAC (Matys et al., 2006), and other motif collections, possibly due to low redundancy in motif position weight matrices.

Bedtools (version 2.25) was used to generate a Markov model of order 1 from all peak intervals to use as a background peak file (parameter "—bgfile"). For each TF, the target set was further refined by filtering out binding sites below the top quartile, ranked by the FIMO p-value. TF binding sites were linked to a target gene if the peak fell within a $1 \mathrm{~kb}$ window of the gene body, creating a prior matrix with 601 columns and 24909 rows (Table S4). Entries at row "I" column "J" were set to 1 to denote a putative regulatory event between TF "i" and target gene "j." All other entries were set to 0 , which penalizes, but does not completely prevent a regulatory edge connecting "i" and "j."

\section{Expression Normalization}

Expression data was normalized using DESeq2 to remove the dependence of the variance on the mean. We explored linear models for batch correction such as ComBat and limma, but these models were ruled out as they superimpose each experiment's center of mass essentially placing different experiments on top of one another in PCA space-experiments 
which should have unique, non-overlapping centers of mass (due to having different ratios of stimulus conditions as compared to mock conditions). Thus, we used DESeq2 rlog- transformed expression data to normalized gene expression values relative to mock conditions for each time point and for each donor by doing a vector subtraction. In our time series RNA-seq experiment, 3 samples were identified as outliers in gene expression biclusters from DESeq2 analysis (noted in Metadata Table S1), consistently clustering away from the rest of the 93 samples across nearly all condition contrasts in PCA plots. Since network inference is sensitive to outliers due to a z-scoring step, and because follow-up RNA-seq experiments under the same conditions supported the idea that these samples were outliers, these 3 samples were removed from the network inference procedure.

\section{Estimation of TF activities}

The network inference methodology used here builds directly upon our previous platform, the Inferelator, in which the log gene expression is modeled as the linear combination of TF predictor variables (Bonneau et al., 2006). Earlier versions of the inferelator model used the TF expression as the predictor, but current methods use a latent variable, the estimated TF activity, as the predictor (Greenfield et al., 2013). The activity of a TF is inferred from the changes of the TF's putative target genes and has been shown to be able to uncover more known relationships between TFs and target genes than using TF expression as the predictor (Arrieta-Ortiz et al., 2015).

In the Inferelator, we define the equation for inferring a network as $X=B A$. " $X$ " is the gene expression matrix, " $\mathrm{A}$ " is the transcription factor activity matrix, and " $\mathrm{B}$ " is the betas matrix, which can also be thought as the connectivity network. If beta is found to be nonzero, then we have inferred an association between the gene and the TF. This linear equation appears straightforward, but it cannot be immediately solved because there are two unknowns, both "B," 
the network beta value, and "A," the TF activity. We therefore use a two-step process. First, we solve for "A," using the prior network, with the equation $X=P A$. In this equation, the prior network "P" is known. However, because the confidence in any one of its chromatin accessibility derived entries is low, we randomly leave out half of the entries of $P$, and do this iteratively 400 times, deriving 400 estimates for the activities, "A." We solve the inverse problem $X=P A$ by multiplying " $X$ " by the pseudoinverse of the prior matrix, " $P$ ". The second step of the process is to solve the linear regression problem $X=B A$, with " $B$ " as the unknown, which we do using stability selection with elastic net regularization.

\section{Network inference with subsampled priors}

Since the prior matrix was built with thresholding at fixed p-values, both at the level of peak finding and the level of motif binding, any single entry in the prior matrix cannot be considered accurate. In order to improve computational reproducibility, we use a subsampling strategy, which was recently pioneered in a different context to increase prediction accuracy when assessing whether pathway perturbations hold true across different animal species (Hafemeister et al., 2015). This subsampling strategy decreases the density of the prior matrix, which is a desideratum when the number of targets per TF in the prior is more than 2000 on average. Therefore, for each of the 400 computational estimations of the transcription factor activity, we sampled $50 \%$ of the nonzero entries in the prior without replacement. This enabled TFs with similar motif preferences and subtle differences in target sets to be teased apart over the course of many Inferelator runs. For example, in a single Inferelator run, a number of different IRF family members could be assigned at random to have undue emphasis on IFN and ISG expression, since many IRFs overlap in motif preference. In some extreme single run cases, due to inherent randomness in the inference procedure, no IRFs were assigned to IFNB1 or IFNL1. In contrast, after multiple runs where estimated TF activities were subsampled, the 
ATAC-seq-based network correctly predicted a strong influence of IRF3 on IFNB1, IFNL1, and core ISGs target sets were teased apart from other IRFs.

\section{Ensemble Model and AUPR curves}

The 400 individual models that were generated by the inferelator were combined by ranking each interaction by summed beta values to generate an ensemble network (EN-ATAC x400). While this ensemble model predicted over 2 million interactions, we have provided a table to accompany this manuscript that lists the top 500,000 edges, selected based on absolute value of the beta sign (Table S5). Networks of this size cannot be reasonably visualized or efficiently analyzed by current computational resources, so for this work we chose to display the top 75,000 edges in the ensemble network. At this choice of a cutoff in ranked interactions, there are 542 TFs assigned as regulators for 21862 targets. This ranking was used to compute an area under the precision-recall curve (AUPR) by validating the predicted interactions against "gold standard" TF-to-gene-target connections that have been reported in the literature.

\section{Proportional Venn Diagrams}

Venn diagrams were generated using BioVenn (Hulsen et al., 2008). IRFs 1, 3, 5, 7, 8, and 9 and the NF-KB family members RELA, RELB, REL, NFKB1, and NFKB2 are known to be important for primary and secondary IFN responses and influence ISG expression. We determined whether the targets of these IRFs and the NF-KB family members (Figure S3E), or specific targets of IRF3 that were predicted by the EN-ATAC $\times 400$ network or the BBSR network (Figure S3F) overlapped with a set of "core" mammalian ISGs (Shaw et al., 2017).

\section{Hypergeometric tests for TF enrichment}

With the network model established, we can then ask whether any given Transcription Factor is associated with differential gene expression through its network targets. For every contrast, we 
used the hypergeometric test for enrichment to query whether the set of differentially expressed genes for a given contrast was enriched for the targets of any of the transcription factors. We used a Bonferroni correction, dividing by the number of transcription factors, when estimating these $p$-values. For finding a global ranking of enrichment across multiple differential gene expression contrasts, the weighted z-method for combining probabilities was used with equal weights following a previously defined method (Whitlock, 2005).

\section{Modularity and similarity K-means clustering}

In order to interpret the results of the network inference, which yielded a network with 75,000 edges, we asked whether there existed large-scale clusters in the networks. To this end we used the python-louvain clustering module, which implements the modularity clustering algorithm, maximizing an objective function that sums edges within communities while subtracting edges between communities (Blondel, 2008). Due to the stochasticity of this algorithm, where the modularity score and even the number of clusters varies with each run, we repeated the run until convergence, which occurred on the order of a thousand runs. This computational approach allowed us not only to create clusters for large-scale communities, but also build a dendogram of pairwise similarities between every gene in the network, where the pairwise entry corresponds to the number of times in the thousand runs that the two genes were clustered together. This dendogram allowed us to identify "unclustered" nodes in the network: genes that do not consistently cluster with any of the main groups. The dendogram was split into 500 , which resulted in 10 major clusters, defined as a cluster with more than $1 \%$ of the total gene set. These clusters were then labeled with identifiers from Enrichr pathway analysis. For complete pathway analysis results for the top 10 clusters, please see:

Cluster 1 http://amp.pharm.mssm.edu/Enrichr/enrich?dataset=3dhb9

Cluster 2 http://amp.pharm.mssm.edu/Enrichr/enrich?dataset=3dhbc 
Cluster 3 http://amp.pharm.mssm.edu/Enrichr/enrich?dataset $=3 \mathrm{dhbo}$

Cluster 4 http://amp.pharm.mssm.edu/Enrichr/enrich?dataset=3dhfq

Cluster 5 http://amp.pharm.mssm.edu/Enrichr/enrich?dataset=3dhgc

Cluster 6 http://amp.pharm.mssm.edu/Enrichr/enrich?dataset=3dhgi

Cluster 7 http://amp.pharm.mssm.edu/Enrichr/enrich?dataset=3dhgn

Cluster 8 http://amp.pharm.mssm.edu/Enrichr/enrich?dataset=3dhgp

Cluster 9 http://amp.pharm.mssm.edu/Enrichr/enrich?dataset=3dhgq

Cluster 10 http://amp.pharm.mssm.edu/Enrichr/enrich?dataset=3dhgr

\section{Network Visualization}

Network visualization software Gephi Version 0.9.1 (Bastian, 2009) was used to visualize the network using the Force Atlas2 layout algorithm (Jacomy et al., 2014). For visualizing smaller network components with louvain clustering, we used a jp_gene_viz visualization tool developed at the Simons Foundation. The Gephi-formatted network file is available upon request.

\section{Gene Set Enrichment Analysis (GSEA)}

GSEA was performed by comparing each condition in the time series RNA-seq data to the corresponding mock time point. 36883 gene features for each condition were ranked by the signal to noise metric of GSEA and the analysis was performed using the standard weighted enrichment statistic against 3815 human gene sets contained in the Molecular Signatures Database that included all $(\mathrm{H})$ Hallmark gene sets, $(\mathrm{C} 2)$ curated gene sets, and $(\mathrm{C} 3)$ motif gene sets. In a first pass analysis, the normalized enrichment score (NES) was calculated using 500 gene set permutations and once relevant gene sets were identified a more stringent statistical analysis was performed using 1000 phenotype permutations. Full GSEA results can be 
accessed through the following link:

https://www.dropbox.com/sh/2u4psikt2tyz4r0/AAB4Re3YKtOA5lqPEEAwfR7Aa?dl=0

\section{Immunoblotting}

1 million cells were lysed in $100 \mu \mathrm{L}$ of RIPA buffer $(50 \mathrm{mM}$ Tris $\mathrm{HCl}, 150 \mathrm{mM} \mathrm{NaCl}, 0.1 \%$ SDS, 0.5\% DOC, 1\% NP-40, Protease inhibitor (Roche; 1187358001)). Lysis was performed on ice for 30 minutes. Lysates were cleared by centrifugation at $8000 \mathrm{~g}$ for 8 minutes at $4{ }^{\circ} \mathrm{C}$, and $20 \mu \mathrm{l}$ of Laemmli 6X (12\% SDS, 30\% Glycerol, 0.375M Tris-HCl pH6.8, 30\% 2-mercaptoehtanol, 1\% bromophenol blue) was added and samples were boiled at $95^{\circ} \mathrm{C}$ for $15 \mathrm{~min}$. Lysates were resolved on Criterion or 4\%-20\% Biorad precast SDS-PAGE gels and transferred on PVDF membrane. Membranes were saturated and proteins were blotted with antibodies (listed in Key resources table) in 5\% non-fat dry milk, PBS, $0.1 \%$ Tween buffer. ECL signal was recorded on the ChemiDoc-XRS or ChemiDoc Touch Biorad Imager. Data was analyzed and quantified with the Image Lab software (Biorad).

\section{Quantitative PCR}

50,000 to 200,000 cells were lysed in TRIzol reagent (Thermo Fisher) and then RNA was isolated following the manufacturer's instructions with minor modifications. In brief, we performed two sequential chloroform extractions and added Glycoblue (Thermo Fisher) as a carrier prior to precipitation with isopropanol. RNA pellets were washed in $75 \%$ ethanol and resuspended in $200 \mu \mathrm{l}$ of DNase and RNase-free water. $500 \mu \mathrm{g}$ of RNA was converted into cDNA using Superscript III (ThermoFisher). Quantitative PCR reactions were carried out using TaqMan primer probes (ABI) and TaqMan Fast Universal PCR Master Mix (ThermoFisher) in either a Lightcycler (Roche) or a CFX96 thermocycler (BioRad) in a volume of $10 \mu$ according to the following cycling conditions: $50{ }^{\circ} \mathrm{C}$ for $2 \mathrm{~min}, 95^{\circ} \mathrm{C}$ for $2 \mathrm{~min}$, then 55 cycles each of $95^{\circ} \mathrm{C}$ for $3 \mathrm{sec}$, to $60^{\circ} \mathrm{C}$ for $30 \mathrm{sec}$, followed by $95^{\circ} \mathrm{C}$ for $5 \mathrm{sec}$. A melting curve analysis was then 
performed going from $65^{\circ} \mathrm{C}$ to $95^{\circ} \mathrm{C}$ in $0.5^{\circ} \mathrm{C}$ intervals every $5 \mathrm{sec}$. Data were plotted as expression relative to GAPDH 1000 .

\section{IFNL1 Protein Quantification}

IFNL1 protein concentrations were measured on supernatants from infected or treated DCs using a LEGENDplex Human Anti-Virus Response assay (BioLegend) according to the manufacturer's protocol. Data were acquired on a BD FACSVerse (BD) and analyzed with LEGENDplex Software (BioLegend).

\section{Bioassays for type I IFN}

To quantify IFN activity from infected or stimulated cells we assayed supernatants with HL116 reported cells that contain firefly luciferase gene under control of the IFN-inducible 6-16 promoter (Uze et al., 1994). Supernatants from treated or untreated DCs were transferred to 20,000 HL116 cells in 96 well plates. After 7 h, HL116 cells were lysed in passive lysis buffer and subsequently scored for luciferase activity using a luciferin-based method (Promega). Relative light units were converted to units per ml of IFN using a standard curve that was generated from serial dilutions of recombinant human IFNa2a, with $\mathrm{HL} 116$ cells responding in a linear range between 2 and $200 \mathrm{U} / \mathrm{ml}$ of IFN.

\section{QUANTIFICATION AND STATISTICAL ANALYSIS}

Statistical analyses incorporated into the computational implementation of network inference were performed as stated above in the STAR METHODS section. Otherwise, statistical tests were performed as indicated in the figure legends or using Prism 6.0 (GraphPad) to calculate either a two-way ANOVA with Sidak's multiple comparisons test or a two-tailed t test using paired samples. The number of unique donors for DC experiments is also listed in the figure 
legends, representing the number of biological replicates performed for a given experiment. Data reflects pooled data from multiple experiments where indicated.

\section{DATA AND SOFTWARE AVAILABILITY}

The RNA-seq data have been deposited in the Gene Expression Omnibus (GEO) database under ID code GSE125817. The ATAC-seq data have been deposited in the GEO database under ID code GSE125918. Both can be accessed from the GEO series code GSE125919.

\section{KEY RESOURCES TABLE}

\begin{tabular}{|c|c|c|}
\hline REAGENT or RESOURCE & SOURCE & IDENTIFIER \\
\hline \multicolumn{3}{|l|}{ Antibodies } \\
\hline Anti-Human CD86 (B7-2) PE-Cyanine5 & eBioscience & $\begin{array}{l}\text { Cat\# 15-0869-42 } \\
\text { RRID:AB_1104200 } \\
3\end{array}$ \\
\hline Anti-Human CD86 (B7-2) PE & Thermo Fisher & $\begin{array}{l}\text { Cat\#12-0869- } \\
\text { 42,RRID: } \\
\text { AB_10732345 }\end{array}$ \\
\hline Anti-Human CD11C-APC & Thermo Fisher & $\begin{array}{l}\text { Cat\# 17-0116-42, } \\
\text { RRID:AB_1659668 }\end{array}$ \\
\hline Anti-Human HLA-DR PE-Cyanine5 & BioLegend & $\begin{array}{l}\text { Cat\# 307607, } \\
\text { RRID:AB_314685 }\end{array}$ \\
\hline Anti-Human DC-SIGN PE & R\&D Systems & $\begin{array}{l}\text { Cat\# FAB161P, } \\
\text { RRID:AB_357064 }\end{array}$ \\
\hline Anti-Human Actin & Millipore & $\begin{array}{l}\text { Cat\# MAB1501, } \\
\text { RRID: } \\
\text { AB_2223041 }\end{array}$ \\
\hline Anti-Human Vinculin (Clone hVIN-1) & Sigma & $\begin{array}{l}\text { Cat\# V9264, } \\
\text { RRID: } \\
\text { AB_10603627 }\end{array}$ \\
\hline Anti-Human PRDM1 Blimp-1 (Clone C14A4) & $\begin{array}{l}\text { Cell Signaling } \\
\text { Technology }\end{array}$ & $\begin{array}{l}\text { Cat\# 9115, RRID: } \\
\text { AB_2169699 }\end{array}$ \\
\hline Anti-Human NONO & Sigma & $\begin{array}{l}\text { Cat\# N8664, } \\
\text { RRID: } \\
\text { AB_10601034 }\end{array}$ \\
\hline True Blot anti-mouse Ig HRP & Thermo Fisher & $\begin{array}{l}\text { Cat\# 18-8817-33, } \\
\text { RRID: } \\
\text { AB_10126892 }\end{array}$ \\
\hline
\end{tabular}




\begin{tabular}{|c|c|c|}
\hline True Blot anti-rabbit Ig HRP & Thermo Fisher & $\begin{array}{l}\text { Cat\# 18-8816- } \\
\text { 31,RRID: } \\
\text { AB_469528 }\end{array}$ \\
\hline \multicolumn{3}{|l|}{ Bacterial and Virus Strains } \\
\hline Stbl3 competent E. coli & Thermo Fisher & Cat\# C737303 \\
\hline \multicolumn{3}{|l|}{ Biological Samples } \\
\hline Human leukocytes from normal donors & $\begin{array}{l}\text { Bloodworks } \\
\text { Northwest }\end{array}$ & $\begin{array}{l}\text { Product code: } \\
2490-03 \\
\text { http://www.bloodw } \\
\text { orksnw.org/ }\end{array}$ \\
\hline Human leukocytes from normal donors & $\begin{array}{l}\text { New York Blood } \\
\text { Center }\end{array}$ & $\mathrm{N} / \mathrm{A}$ \\
\hline Human leukocytes from normal donors & $\begin{array}{l}\text { ARUP Blood } \\
\text { Services }\end{array}$ & N/A \\
\hline Human leukocytes from normal donors & $\begin{array}{l}\text { venipunctures } \\
\text { approved by the } \\
\text { Institut National de } \\
\text { la Santé et de la } \\
\text { Recherche Médicale } \\
\text { ethics committee, } \\
\text { Paris, France }\end{array}$ & N/A \\
\hline \multicolumn{3}{|c|}{ Chemicals, Peptides, and Recombinant Proteins } \\
\hline Ficoll-Paque Plus & GE Healthcare & Cat\# 17-1440-02 \\
\hline Fetal Bovine Serum & Peak Serum Inc & (Lot\#125N16) \\
\hline Fetal Bovine Serum & Gibco & (Lot \#1982147) \\
\hline Bovine Serum Albumin & Roche & Cat\# 03116956001 \\
\hline Recombinant Human IL-4 & Preprotech & Cat\# 200-04 \\
\hline Recombinant Human IL-4 & Miltenyi Biotech & Cat\# 130-093-922 \\
\hline Recombinant Human GM-CSF & Preprotech & Cat\# 300-03 \\
\hline Recombinant Human GM-CSF & Miltenyi Biotech & Cat\# 130-093-867 \\
\hline HEPES & Sigma & Cat\# H3537 \\
\hline Phosphate Buffer Saline (PBS) & Corning & Cat\# 45000-446 \\
\hline EDTA $0.5 \mathrm{M}$ & Sigma & Cat\# E7889 \\
\hline Penicillin-Streptomycin & Thermo Fisher & Cat\# 15140-122 \\
\hline 2-Mercaptoethanol & Thermo Fisher & Cat\# 21985023 \\
\hline L-glutamine & Thermo Fisher & Cat\# 25030081 \\
\hline MEM non-essential amino acids & Thermo Fisher & Cat\# 11140050 \\
\hline Sodium Pyruvate & Thermo Fisher & Cat\# 11360070 \\
\hline RPMI 1640 medium & Thermo Fisher & Cat\# 11875-119 \\
\hline DMEM & Thermo Fisher & Cat\# 11965-118 \\
\hline HAT supplement & Thermo Fisher & Cat\# 21060-017 \\
\hline poly-L-lysine hydrobromide & MP Biomedicals & Cat\# 0219454405 \\
\hline
\end{tabular}




\begin{tabular}{|c|c|c|}
\hline $\begin{array}{l}\text { polyl:C high molecular weight } \\
\text { Penicillin-Streptomycin }\end{array}$ & InvivoGen & Cat\# tlrl-pic \\
\hline 2'3'-cGAMP (cyclic [G(2',5')pA(3',5')p] & InvivoGen & Cat\# tIrl-cga23-s \\
\hline Recombinant Human Interferon-alpha 2 alpha & Thermo Fisher & Cat \# 111001 \\
\hline Recombinant Human Interferon-alpha 2 alpha & ImmunoTools & $\begin{array}{l}\text { Cat\# 11343506, } \\
\text { (No CAS) }\end{array}$ \\
\hline LPS - ultra pure from Salmonella minnesota R595 & $\begin{array}{l}\text { List Biological } \\
\text { Laboratories, INC. }\end{array}$ & Cat\# 434 \\
\hline R848 & Invivogen & $\begin{array}{l}\text { Cat\# t|rl-r848, } \\
\text { CAS: } 144875-48-9\end{array}$ \\
\hline Poly(dG:dC) & Invivogen & $\begin{array}{l}\text { Cat\# tlrl-pgcn, } \\
\text { CAS: } 90385-88-9\end{array}$ \\
\hline Cyclosporine A & Euromedex & $\begin{array}{l}\text { Cat\# S2286; CAS: } \\
\text { 59865-13-3 }\end{array}$ \\
\hline Am80 & Tocris & Cat\# 3507 \\
\hline BMS 195614 & Tocris & Cat\# 3660 \\
\hline all-trans retinoic acid (ATRA) & Tocris & Cat\# 0695 \\
\hline TRIzol reagent & Thermo Fisher & Cat\# 15596026 \\
\hline GlycoBlue Coprecipitate & Thermo Fisher & Cat\# AM9516 \\
\hline $\begin{array}{l}\text { Polyethylenimine "Max", (Mw 40,000) - High } \\
\text { Potency Linear PEI }\end{array}$ & Polysciences, Inc. & Cat\# 9002-98-6 \\
\hline Paraformaldehyde & $\begin{array}{l}\text { Electron Microscopy } \\
\text { Sciences }\end{array}$ & Cat\# 15713-S \\
\hline Polybrene & Sigma & Cat\# TR-1003-G \\
\hline Puromycin & Invivogen & Cat\# ant-pr-1 \\
\hline TaqMan Fast Universal PCR Master Mix & Thermo Fisher & Cat\# 4352402 \\
\hline NEBNext® High-Fidelity 2X PCR Master Mix & $\begin{array}{l}\text { New England } \\
\text { Biolabs }\end{array}$ & Cat\# M0541S \\
\hline SYBR Green I Nucleic Acid Stain & Thermo Fisher & Cat\# S-7563 \\
\hline $\begin{array}{l}\text { cOmplete, EDTA-free, Protease inhibitor cocktails } \\
\text { tablets }\end{array}$ & Roche & Cat\# 11873580001 \\
\hline \multicolumn{3}{|l|}{ Critical Commercial Assays } \\
\hline LIVE/DEAD® Fixable Violet Dead Cell Stain Kit & Molecular Probes & Cat\# L34955 \\
\hline $\begin{array}{l}\text { Complete kit (Universal) KAPA Library } \\
\text { Quantification Kits for Next-Generation Sequencing }\end{array}$ & Kappa Biosystems & Cat\# KK4824 \\
\hline Nextera DNA Library Prep Kit & Illumina Inc. & Cat\# FC-121-1030 \\
\hline Plasmid DNA Purification Nucleobond Xtra Maxi Kit & Takara & Cat\# 740414.100 \\
\hline DNeasy Blood \& Tissue Kit & Qiagen & Cat\# 69504 \\
\hline Minelute Kit & Qiagen & Cat\# 28004 \\
\hline Luciferase assay system & Promega & Cat\# E4530 \\
\hline $\begin{array}{l}\text { LEGENDplex Human Type 1/2/3 Interferon Panel } \\
\text { (5-plex) }\end{array}$ & Ozyme & Cat\# BLE740396B \\
\hline \multicolumn{3}{|l|}{ Deposited Data } \\
\hline RNA-seq on stimulated DCs & This paper & GSE125817 \\
\hline
\end{tabular}




\begin{tabular}{|c|c|c|}
\hline ATAC-seq on stimulated DCs & This paper & GSE125918 \\
\hline \multicolumn{3}{|l|}{ Experimental Models: Cell Lines } \\
\hline HEK 293FT cells & Thermo Fisher & $\begin{array}{l}\text { Cat\# R70007, } \\
\text { RRID:CVCL_6911 }\end{array}$ \\
\hline HL116 reporter cells & (Uze et al., 1994) & $\begin{array}{l}\text { Gift from Sandra } \\
\text { Pellegrini }\end{array}$ \\
\hline \multicolumn{3}{|l|}{ Experimental Models: Organisms/Strains } \\
\hline $\mathrm{N} / \mathrm{A}$ & $\mathrm{N} / \mathrm{A}$ & $\mathrm{N} / \mathrm{A}$ \\
\hline \multicolumn{3}{|l|}{ Oligonucleotides } \\
\hline ATAC-seq sequencing primers & $\begin{array}{l}\text { (Buenrostro et al., } \\
\text { 2013) }\end{array}$ & (see Table S7) \\
\hline TaqMan GAPDH assay & ThermoFisher & $\begin{array}{l}\text { Cat\# } \\
\text { Hs02758991_g1 }\end{array}$ \\
\hline TaqMan ISG15 assay & ThermoFisher & $\begin{array}{l}\text { Cat\# } \\
\text { Hs01921425_s1 }\end{array}$ \\
\hline TaqMan CXCL10 assay & ThermoFisher & $\begin{array}{l}\text { Cat\# } \\
\text { Hs01124251_g1 }\end{array}$ \\
\hline TaqMan cGAS/MB21D1 assay & ThermoFisher & $\begin{array}{l}\text { Cat\# } \\
\text { Hs00403553_m1 }\end{array}$ \\
\hline TaqMan eGFP assay & ThermoFisher & $\begin{array}{l}\text { Cat\# } \\
\text { Mr04329676_mr }\end{array}$ \\
\hline TaqMan IRF3 assay & ThermoFisher & $\begin{array}{l}\text { Cat\# } \\
\text { Hs01547283_m1 }\end{array}$ \\
\hline TaqMan HIVEP1 assay & ThermoFisher & $\begin{array}{l}\text { Cat\# } \\
\text { Hs00172428_m1 }\end{array}$ \\
\hline TaqMan KLF13 assay & ThermoFisher & $\begin{array}{l}\text { Cat\# } \\
\text { Hs00429818_m1 }\end{array}$ \\
\hline TaqMan RARA assay & ThermoFisher & $\begin{array}{l}\text { Cat\# } \\
\text { Hs00940446_m1 }\end{array}$ \\
\hline TaqMan STAT2 assay & ThermoFisher & $\begin{array}{l}\text { Cat\# } \\
\text { Hs01013123_m1 }\end{array}$ \\
\hline TaqMan IFNB1 assay & ThermoFisher & $\begin{array}{l}\text { Cat\# } \\
\text { Hs01077958_s1 }\end{array}$ \\
\hline TaqMan IFNL1 assay & ThermoFisher & $\begin{array}{l}\text { Cat\# } \\
\text { Hs00601677_g1 }\end{array}$ \\
\hline \multicolumn{3}{|l|}{ Recombinant DNA } \\
\hline HIV-1-GFP & (Manel et al., 2010) & $\begin{array}{l}\text { HIV-1 GFP- } \\
\text { reporter virus }\end{array}$ \\
\hline HIV-2-GFP & (Manel et al., 2010) & $\begin{array}{l}\text { HIV-2 GFP- } \\
\text { reporter virus }\end{array}$ \\
\hline LKO-GFP & (Manel et al., 2010) & $\begin{array}{l}\text { Non-replicating } \\
\text { lentivector }\end{array}$ \\
\hline P86-HA & (Lahaye et al., 2013) & $\begin{array}{l}\text { Mutagenized virus } \\
\text { HIVac-2 }\end{array}$ \\
\hline $\mathrm{NL}(\mathrm{AD} 8)$ & (Manel et al., 2010) & $\begin{array}{l}\text { wild type HIV-1 } \\
\text { molecular clone }\end{array}$ \\
\hline JK7312AS & $\begin{array}{l}\text { Gift from Beatrice } \\
\text { Hahn (Lahaye et al., } \\
\text { 2018) }\end{array}$ & $\begin{array}{l}\text { wild type HIV-2 } \\
\text { molecular clone }\end{array}$ \\
\hline pSIV3+ (for production of virus-like particles & (Mangeot et al., & SIVmac251 \\
\hline
\end{tabular}




\begin{tabular}{|c|c|c|}
\hline containing Vpx) & 2000) & $\begin{array}{l}\text { GenBank acc. no. } \\
\text { M19499 }\end{array}$ \\
\hline pLKO.1 - TRC cloning vector & $\begin{array}{l}\text { Gift from David Root } \\
\text { (Moffat et al., 2006) }\end{array}$ & $\begin{array}{l}\text { Addgene plasmid } \\
\# 10878\end{array}$ \\
\hline psPAX2 & $\begin{array}{l}\text { Gift from Didier } \\
\text { Trono }\end{array}$ & $\begin{array}{l}\text { Addgene plasmid } \\
\# 12260\end{array}$ \\
\hline pCMV-VSV-G & $\begin{array}{l}\text { Gift from Bob } \\
\text { Weinberg (Stewart } \\
\text { et al., 2003) }\end{array}$ & $\begin{array}{l}\text { Addgene plasmid } \\
\# 8454\end{array}$ \\
\hline control shRNA & Sigma & SHC002 \\
\hline LacZ shRNA & Sigma & TRCN0000072229 \\
\hline NONO shRNA & Sigma & TRCN0000074562 \\
\hline cGas shRNA & Sigma & TRCN0000149984 \\
\hline IRF3 sh & $\begin{array}{l}\text { (Johnson et al., } \\
\text { 2018) }\end{array}$ & N/A \\
\hline HIVEP1 sh1 & Sigma & TRCN0000235474 \\
\hline HIVEP1 sh3 & Sigma & TRCN0000235476 \\
\hline KLF13 sh1 & Sigma & TRCN0000016923 \\
\hline KLF13 sh2 & Sigma & TRCN0000016925 \\
\hline STAT2 sh1 & Sigma & TRCN0000364399 \\
\hline STAT2 sh2 & Sigma & TRCN0000364400 \\
\hline RARA sh1 & Sigma & TRCN0000275554 \\
\hline RARA sh2 & Sigma & TRCN0000020370 \\
\hline PRDM1 sh2 & Sigma & TRCN0000013609 \\
\hline PRDM1 sh3 & Sigma & TRCN0000013610 \\
\hline PRDM1 sh5 & Sigma & TRCN0000013612 \\
\hline \multicolumn{3}{|l|}{ Software and Algorithms } \\
\hline $\mathrm{R}$ & $\mathrm{N} / \mathrm{A}$ & $\begin{array}{l}\text { https://www.r- } \\
\text { project.org/ }\end{array}$ \\
\hline Integrative Genomics Viewer (IGV) & $\mathrm{N} / \mathrm{A}$ & $\begin{array}{l}\text { http://software.bro } \\
\text { adinstitute.org/soft } \\
\text { ware/igv/ }\end{array}$ \\
\hline FlowJo Version 8.7 & FlowJo LLC & $\begin{array}{l}\text { https://www.flowjo. } \\
\text { com/ }\end{array}$ \\
\hline FlowJo Version 9.9.4 & FlowJo LLC & $\begin{array}{l}\text { https://www. flowjo. } \\
\text { com/ }\end{array}$ \\
\hline LEGENDplex Software - Version 8.0 & LEGENDplex & $\begin{array}{l}\text { http://www.vigenet } \\
\text { ech.com/LEGEND } \\
\text { plex7.htm }\end{array}$ \\
\hline Image Lab software - Version 5.2.1 & BioRad & $\begin{array}{l}\text { http://www.bio- } \\
\text { rad.com/fr- } \\
\text { fr/product/image- } \\
\text { lab- } \\
\text { software?ID=KRE } \\
6 \text { P5E8Z }\end{array}$ \\
\hline GraphPad Prism 6.0 & GraphPad & $\begin{array}{l}\text { https://www.graph } \\
\text { pad.com/scientific- } \\
\text { software/prism/ }\end{array}$ \\
\hline
\end{tabular}




\begin{tabular}{|c|c|c|}
\hline Enrichr & $\begin{array}{l}\text { (Chen et al., 2013; } \\
\text { Kuleshov et al., } \\
2016)\end{array}$ & $\begin{array}{l}\text { http://amp.pharm. } \\
\text { mssm.edu/Enrichr/ }\end{array}$ \\
\hline Inferelator & $\begin{array}{l}\text { (Bonneau et al., } \\
2006 \text { ) }\end{array}$ & $\mathrm{N} / \mathrm{A}$ \\
\hline Gephi & (Bastian, 2009) & https://gephi.org/ \\
\hline BioVenn & (Hulsen et al., 2008) & $\begin{array}{l}\text { http://www.biovenn } \\
. \mathrm{nl} /\end{array}$ \\
\hline \multicolumn{3}{|l|}{ Other } \\
\hline LS Columns & Miltenyi Biotec, Inc & $130-042-401$ \\
\hline SW 28 Ti Rotor, Swinging Bucket & Beckman & Cat\# 342204 \\
\hline Thinwall Polyallomer, Conical Tubes; Size:25 x 89 & Beckman & Cat\# 358126 \\
\hline $0.45 \mu \mathrm{m}$ syringe filters & Corning & Cat\# 28200-026 \\
\hline QuadroMACS Separator & Miltenyi Biotec, Inc & $130-090-976$ \\
\hline CD14 MicroBeads, human & Miltenyi Biotec, Inc & $130-050-201$ \\
\hline LSR II Flow Cytometer & BD Biosciences & $\mathrm{N} / \mathrm{A}$ \\
\hline FACSAria II Cell Sorter & BD Biosciences & N/A \\
\hline CFX96 thermal cycler & Biorad & Model T100 \\
\hline Lightcycler 480 System & Roche & $\begin{array}{l}\text { Product\# } \\
05015278001\end{array}$ \\
\hline
\end{tabular}

Excel Tables not appended to the Supplemental PDF:

Table S2. Expression matrix. Related to Figure 1.

Table S3. ATAC-seq peaks. Related to Figure 2.

Table S4. Prior matrix. Related to Figure 3.

Table S5. DC gene regulatory network (EN-ATAC x400) capped at 500k edges. Related to Figure 4.

Table S6. TF Hypergeometric enrichment. Related to Figure 4. 


\section{References}

Amit, I., Garber, M., Chevrier, N., Leite, A.P., Donner, Y., Eisenhaure, T., Guttman, M., Grenier, J.K., Li, W., Zuk, O., et al. (2009). Unbiased reconstruction of a mammalian transcriptional network mediating pathogen responses. Science 326, 257-263.

Arrieta-Ortiz, M.L., Hafemeister, C., Bate, A.R., Chu, T., Greenfield, A., Shuster, B., Barry, S.N., Gallitto, M., Liu, B., Kacmarczyk, T., et al. (2015). An experimentally supported model of the Bacillus subtilis global transcriptional regulatory network. Molecular systems biology 11, 839.

Banchereau, J., Briere, F., Caux, C., Davoust, J., Lebecque, S., Liu, Y.J., Pulendran, B., and Palucka, K. (2000). Immunobiology of dendritic cells. Annual review of immunology 18, 767811.

Bastian, M.H., S.; Jacomy, M. (2009). Gephi: an open source software for exploring and manipulating networks. International AAAI Conference on Weblogs and Social Media.

Blondel, V.D.G., J.; Lambiotte, R.; Lefebvre, E. (2008). Fast unfolding of communities in large networks. J Stat Mech, P10008.

Bonelt, P., Wohner, M., Minnich, M., Tagoh, H., Fischer, M., Jaritz, M., Kavirayani, A., Garimella, M., Karlsson, M.C., and Busslinger, M. (2019). Precocious expression of Blimp1 in B cells causes autoimmune disease with increased self-reactive plasma cells. EMBO J 38.

Bonneau, R., Reiss, D.J., Shannon, P., Facciotti, M., Hood, L., Baliga, N.S., and Thorsson, V. (2006). The Inferelator: an algorithm for learning parsimonious regulatory networks from systems-biology data sets de novo. Genome Biol 7, R36.

Buenrostro, J.D., Giresi, P.G., Zaba, L.C., Chang, H.Y., and Greenleaf, W.J. (2013).

Transposition of native chromatin for fast and sensitive epigenomic profiling of open chromatin, DNA-binding proteins and nucleosome position. Nat Methods 10, 1213-1218.

Cao, X. (2016). Self-regulation and cross-regulation of pattern-recognition receptor signalling in health and disease. Nature reviews Immunology 16, 35-50.

Chen, E.Y., Tan, C.M., Kou, Y., Duan, Q., Wang, Z., Meirelles, G.V., Clark, N.R., and Ma'ayan, A. (2013). Enrichr: interactive and collaborative HTML5 gene list enrichment analysis tool. BMC Bioinformatics 14, 128.

Chevrier, N., Mertins, P., Artyomov, M.N., Shalek, A.K., Iannacone, M., Ciaccio, M.F., GatViks, I., Tonti, E., DeGrace, M.M., Clauser, K.R., et al. (2011). Systematic discovery of TLR signaling components delineates viral-sensing circuits. Cell 147, 853-867.

Ciofani, M., Madar, A., Galan, C., Sellars, M., Mace, K., Pauli, F., Agarwal, A., Huang, W., Parkhurst, C.N., Muratet, M., et al. (2012). A validated regulatory network for Th17 cell specification. Cell 151, 289-303. 
Corces, M.R., Granja, J.M., Shams, S., Louie, B.H., Seoane, J.A., Zhou, W., Silva, T.C., Groeneveld, C., Wong, C.K., Cho, S.W., et al. (2018). The chromatin accessibility landscape of primary human cancers. Science 362 .

Corrales, L., Matson, V., Flood, B., Spranger, S., and Gajewski, T.F. (2017). Innate immune signaling and regulation in cancer immunotherapy. Cell research 27, 96-108.

Cusanovich, D.A., Hill, A.J., Aghamirzaie, D., Daza, R.M., Pliner, H.A., Berletch, J.B., Filippova, G.N., Huang, X., Christiansen, L., DeWitt, W.S., et al. (2018). A Single-Cell Atlas of In Vivo Mammalian Chromatin Accessibility. Cell 174, 1309-1324 e1318.

Deeks, S.G., Tracy, R., and Douek, D.C. (2013). Systemic effects of inflammation on health during chronic HIV infection. Immunity 39, 633-645.

Delescluse, C., Cavey, M.T., Martin, B., Bernard, B.A., Reichert, U., Maignan, J., Darmon, M., and Shroot, B. (1991). Selective high affinity retinoic acid receptor alpha or beta-gamma ligands. Mol Pharmacol 40, 556-562.

DePaolo, R.W., Abadie, V., Tang, F., Fehlner-Peach, H., Hall, J.A., Wang, W., Marietta, E.V., Kasarda, D.D., Waldmann, T.A., Murray, J.A., et al. (2011). Co-adjuvant effects of retinoic acid and IL-15 induce inflammatory immunity to dietary antigens. Nature 471, 220-224.

Fernandez, S., Tanaskovic, S., Helbig, K., Rajasuriar, R., Kramski, M., Murray, J.M., Beard, M., Purcell, D., Lewin, S.R., Price, P., et al. (2011). CD4+ T-cell deficiency in HIV patients responding to antiretroviral therapy is associated with increased expression of interferonstimulated genes in CD4+ T cells. J Infect Dis 204, 1927-1935.

Gao, D., Wu, J., Wu, Y.T., Du, F., Aroh, C., Yan, N., Sun, L., and Chen, Z.J. (2013). Cyclic GMP-AMP synthase is an innate immune sensor of HIV and other retroviruses. Science 341, 903-906.

Geissmann, F., Revy, P., Brousse, N., Lepelletier, Y., Folli, C., Durandy, A., Chambon, P., and Dy, M. (2003). Retinoids regulate survival and antigen presentation by immature dendritic cells. The Journal of experimental medicine 198, 623-634.

Gilchrist, M., Thorsson, V., Li, B., Rust, A.G., Korb, M., Roach, J.C., Kennedy, K., Hai, T., Bolouri, H., and Aderem, A. (2006). Systems biology approaches identify ATF3 as a negative regulator of Toll-like receptor 4. Nature 441, 173-178.

Goubau, D., Deddouche, S., and Reis e Sousa, C. (2013). Cytosolic sensing of viruses. Immunity $38,855-869$.

Goujon, C., Jarrosson-Wuilleme, L., Bernaud, J., Rigal, D., Darlix, J.L., and Cimarelli, A. (2006). With a little help from a friend: increasing HIV transduction of monocyte-derived dendritic cells with virion-like particles of SIV(MAC). Gene Ther 13, 991-994.

Granelli-Piperno, A., Golebiowska, A., Trumpfheller, C., Siegal, F.P., and Steinman, R.M. (2004). HIV-1-infected monocyte-derived dendritic cells do not undergo maturation but can 
elicit IL-10 production and T cell regulation. Proceedings of the National Academy of Sciences of the United States of America 101, 7669-7674.

Greenfield, A., Hafemeister, C., and Bonneau, R. (2013). Robust data-driven incorporation of prior knowledge into the inference of dynamic regulatory networks. Bioinformatics 29, 10601067.

Greenfield, A., Madar, A., Ostrer, H., and Bonneau, R. (2010). DREAM4: Combining genetic and dynamic information to identify biological networks and dynamical models. PLoS One 5, e13397.

Hafemeister, C., Romero, R., Bilal, E., Meyer, P., Norel, R., Rhrissorrakrai, K., Bonneau, R., and Tarca, A.L. (2015). Inter-species pathway perturbation prediction via data-driven detection of functional homology. Bioinformatics 31, 501-508.

Han, H., Cho, J.W., Lee, S., Yun, A., Kim, H., Bae, D., Yang, S., Kim, C.Y., Lee, M., Kim, E., et al. (2018). TRRUST v2: an expanded reference database of human and mouse transcriptional regulatory interactions. Nucleic Acids Res 46, D380-D386.

Heneka, M.T., Kummer, M.P., and Latz, E. (2014). Innate immune activation in neurodegenerative disease. Nature reviews Immunology 14, 463-477.

Hrecka, K., Hao, C., Gierszewska, M., Swanson, S.K., Kesik-Brodacka, M., Srivastava, S., Florens, L., Washburn, M.P., and Skowronski, J. (2011). Vpx relieves inhibition of HIV-1 infection of macrophages mediated by the SAMHD1 protein. Nature 474, 658-661.

Hulsen, T., de Vlieg, J., and Alkema, W. (2008). BioVenn - a web application for the comparison and visualization of biological lists using area-proportional Venn diagrams. BMC Genomics 9, 488.

Iijima, N., Thompson, J.M., and Iwasaki, A. (2008). Dendritic cells and macrophages in the genitourinary tract. Mucosal Immunol 1, 451-459.

Ivashkiv, L.B., and Donlin, L.T. (2014). Regulation of type I interferon responses. Nature reviews Immunology 14, 36-49.

Iwasaki, A. (2012). A virological view of innate immune recognition. Annual review of microbiology 66, 177-196.

Iwasaki, A., and Medzhitov, R. (2015). Control of adaptive immunity by the innate immune system. Nature immunology 16, 343-353.

Jacomy, M., Venturini, T., Heymann, S., and Bastian, M. (2014). ForceAtlas2, a continuous graph layout algorithm for handy network visualization designed for the Gephi software. PLoS One 9, e98679.

Jankowski, A., Tiuryn, J., and Prabhakar, S. (2016). Romulus: robust multi-state identification of transcription factor binding sites from DNase-seq data. Bioinformatics 32, 2419-2426. 
Johnson, J.S., Lucas, S.Y., Amon, L.M., Skelton, S., Nazitto, R., Carbonetti, S., Sather, D.N., Littman, D.R., and Aderem, A. (2018). Reshaping of the Dendritic Cell Chromatin Landscape and Interferon Pathways during HIV Infection. Cell Host Microbe 23, 366-381 e369.

Jonsson, K.L., Laustsen, A., Krapp, C., Skipper, K.A., Thavachelvam, K., Hotter, D., Egedal, J.H., Kjolby, M., Mohammadi, P., Prabakaran, T., et al. (2017). IFI16 is required for DNA sensing in human macrophages by promoting production and function of cGAMP. Nat Commun 8,14391 .

Kane, M., Zang, T.M., Rihn, S.J., Zhang, F., Kueck, T., Alim, M., Schoggins, J., Rice, C.M., Wilson, S.J., and Bieniasz, P.D. (2016). Identification of Interferon-Stimulated Genes with Antiretroviral Activity. Cell Host Microbe 20,392-405.

Keller, A.D., and Maniatis, T. (1991). Identification and characterization of a novel repressor of beta-interferon gene expression. Genes Dev 5, 868-879.

Ko, Y.A., Chan, Y.H., Liu, C.H., Liang, J.J., Chuang, T.H., Hsueh, Y.P., Lin, Y.L., and Lin, K.I. (2018). Blimp-1-Mediated Pathway Promotes Type I IFN Production in Plasmacytoid Dendritic Cells by Targeting to Interleukin-1 Receptor-Associated Kinase M. Frontiers in immunology 9 , 1828.

Kulakovskiy, I.V., Medvedeva, Y.A., Schaefer, U., Kasianov, A.S., Vorontsov, I.E., Bajic, V.B., and Makeev, V.J. (2013). HOCOMOCO: a comprehensive collection of human transcription factor binding sites models. Nucleic Acids Res 41, D195-202.

Kuleshov, M.V., Jones, M.R., Rouillard, A.D., Fernandez, N.F., Duan, Q., Wang, Z., Koplev, S., Jenkins, S.L., Jagodnik, K.M., Lachmann, A., et al. (2016). Enrichr: a comprehensive gene set enrichment analysis web server 2016 update. Nucleic Acids Res 44, W90-97.

Laguette, N., Sobhian, B., Casartelli, N., Ringeard, M., Chable-Bessia, C., Segeral, E., Yatim, A., Emiliani, S., Schwartz, O., and Benkirane, M. (2011). SAMHD1 is the dendritic- and myeloid-cell-specific HIV-1 restriction factor counteracted by Vpx. Nature 474, 654-657.

Lahaye, X., Gentili, M., Silvin, A., Conrad, C., Picard, L., Jouve, M., Zueva, E., Maurin, M., Nadalin, F., Knott, G.J., et al. (2018). NONO Detects the Nuclear HIV Capsid to Promote cGAS-Mediated Innate Immune Activation. Cell 175, 488-501 e422.

Lahaye, X., Satoh, T., Gentili, M., Cerboni, S., Conrad, C., Hurbain, I., El Marjou, A., Lacabaratz, C., Lelievre, J.D., and Manel, N. (2013). The capsids of HIV-1 and HIV-2 determine immune detection of the viral cDNA by the innate sensor cGAS in dendritic cells. Immunity 39 , 1132-1142.

Lee, M.N., Roy, M., Ong, S.E., Mertins, P., Villani, A.C., Li, W., Dotiwala, F., Sen, J., Doench, J.G., Orzalli, M.H., et al. (2013). Identification of regulators of the innate immune response to cytosolic DNA and retroviral infection by an integrative approach. Nature immunology 14, 179185. 
Levy, D.E., Marie, I.J., and Durbin, J.E. (2011). Induction and function of type I and III interferon in response to viral infection. Current opinion in virology 1, 476-486.

Madar, A., Greenfield, A., Vanden-Eijnden, E., and Bonneau, R. (2010). DREAM3: network inference using dynamic context likelihood of relatedness and the inferelator. PLoS One 5, e9803.

Maldonado, R.A., and von Andrian, U.H. (2010). How tolerogenic dendritic cells induce regulatory T cells. Adv Immunol 108, 111-165.

Manel, N., Hogstad, B., Wang, Y., Levy, D.E., Unutmaz, D., and Littman, D.R. (2010). A cryptic sensor for HIV-1 activates antiviral innate immunity in dendritic cells. Nature 467, 214217.

Mangeot, P.E., Negre, D., Dubois, B., Winter, A.J., Leissner, P., Mehtali, M., Kaiserlian, D., Cosset, F.L., and Darlix, J.L. (2000). Development of minimal lentivirus vectors derived from simian immunodeficiency virus (SIVmac251) and their use for gene transfer into human dendritic cells. Journal of virology 74, 8307-8315.

Mathelier, A., Fornes, O., Arenillas, D.J., Chen, C.Y., Denay, G., Lee, J., Shi, W., Shyr, C., Tan, G., Worsley-Hunt, R., et al. (2016). JASPAR 2016: a major expansion and update of the openaccess database of transcription factor binding profiles. Nucleic Acids Res 44, D110-115.

Matys, V., Kel-Margoulis, O.V., Fricke, E., Liebich, I., Land, S., Barre-Dirrie, A., Reuter, I., Chekmenev, D., Krull, M., Hornischer, K., et al. (2006). TRANSFAC and its module TRANSCompel: transcriptional gene regulation in eukaryotes. Nucleic Acids Res 34, D108-110.

Milner, J.J., Toma, C., Yu, B., Zhang, K., Omilusik, K., Phan, A.T., Wang, D., Getzler, A.J., Nguyen, T., Crotty, S., et al. (2017). Runx3 programs CD8(+) T cell residency in non-lymphoid tissues and tumours. Nature 552, 253-257.

Miraldi, E.R., Pokrovskii, M., Watters, A., Castro, D.M., De Veaux, N., Hall, J.A., Lee, J.Y., Ciofani, M., Madar, A., Carriero, N., et al. (2019). Leveraging chromatin accessibility for transcriptional regulatory network inference in T Helper 17 Cells. Genome Res.

Moffat, J., Grueneberg, D.A., Yang, X., Kim, S.Y., Kloepfer, A.M., Hinkle, G., Piqani, B., Eisenhaure, T.M., Luo, B., Grenier, J.K., et al. (2006). A lentiviral RNAi library for human and mouse genes applied to an arrayed viral high-content screen. Cell 124, 1283-1298.

Nutt, S.L., Fairfax, K.A., and Kallies, A. (2007). BLIMP1 guides the fate of effector B and T cells. Nature reviews Immunology 7, 923-927.

Ramsey, S.A., Klemm, S.L., Zak, D.E., Kennedy, K.A., Thorsson, V., Li, B., Gilchrist, M., Gold, E.S., Johnson, C.D., Litvak, V., et al. (2008). Uncovering a macrophage transcriptional program by integrating evidence from motif scanning and expression dynamics. PLoS Comput Biol 4, e1000021. 
Sandler, N.G., Bosinger, S.E., Estes, J.D., Zhu, R.T., Tharp, G.K., Boritz, E., Levin, D., Wijeyesinghe, S., Makamdop, K.N., del Prete, G.Q., et al. (2014). Type I interferon responses in rhesus macaques prevent SIV infection and slow disease progression. Nature 511, 601-605.

Schoggins, J.W., Wilson, S.J., Panis, M., Murphy, M.Y., Jones, C.T., Bieniasz, P., and Rice, C.M. (2011). A diverse range of gene products are effectors of the type I interferon antiviral response. Nature 472, 481-485.

Shaw, A.E., Hughes, J., Gu, Q., Behdenna, A., Singer, J.B., Dennis, T., Orton, R.J., Varela, M., Gifford, R.J., Wilson, S.J., et al. (2017). Fundamental properties of the mammalian innate immune system revealed by multispecies comparison of type I interferon responses. PLoS biology 15 , e2004086.

Siahpirani, A.F., and Roy, S. (2017). A prior-based integrative framework for functional transcriptional regulatory network inference. Nucleic Acids Res 45, e21.

Smed-Sorensen, A., Lore, K., Vasudevan, J., Louder, M.K., Andersson, J., Mascola, J.R., Spetz, A.L., and Koup, R.A. (2005). Differential susceptibility to human immunodeficiency virus type 1 infection of myeloid and plasmacytoid dendritic cells. Journal of virology 79, 8861-8869.

Stewart, S.A., Dykxhoorn, D.M., Palliser, D., Mizuno, H., Yu, E.Y., An, D.S., Sabatini, D.M., Chen, I.S., Hahn, W.C., Sharp, P.A., et al. (2003). Lentivirus-delivered stable gene silencing by RNAi in primary cells. RNA 9, 493-501.

Subramanian, A., Tamayo, P., Mootha, V.K., Mukherjee, S., Ebert, B.L., Gillette, M.A., Paulovich, A., Pomeroy, S.L., Golub, T.R., Lander, E.S., et al. (2005). Gene set enrichment analysis: a knowledge-based approach for interpreting genome-wide expression profiles. Proceedings of the National Academy of Sciences of the United States of America 102, 1554515550.

Szatmari, I., and Nagy, L. (2008). Nuclear receptor signalling in dendritic cells connects lipids, the genome and immune function. EMBO J 27, 2353-2362.

Szklarczyk, D., Franceschini, A., Wyder, S., Forslund, K., Heller, D., Huerta-Cepas, J., Simonovic, M., Roth, A., Santos, A., Tsafou, K.P., et al. (2015). STRING v10: protein-protein interaction networks, integrated over the tree of life. Nucleic Acids Res 43, D447-452.

Thery, C., and Amigorena, S. (2001). The cell biology of antigen presentation in dendritic cells. Current opinion in immunology $13,45-51$.

Uze, G., Di Marco, S., Mouchel-Vielh, E., Monneron, D., Bandu, M.T., Horisberger, M.A., Dorques, A., Lutfalla, G., and Mogensen, K.E. (1994). Domains of interaction between alpha interferon and its receptor components. J Mol Biol 243, 245-257.

Wada, J., and Makino, H. (2016). Innate immunity in diabetes and diabetic nephropathy. Nature reviews Nephrology 12, 13-26. 
Walker, B., and McMichael, A. (2012). The T-cell response to HIV. Cold Spring Harb Perspect Med 2.

Wang, G.P., Ciuffi, A., Leipzig, J., Berry, C.C., and Bushman, F.D. (2007). HIV integration site selection: analysis by massively parallel pyrosequencing reveals association with epigenetic modifications. Genome Res 17, 1186-1194.

Weirauch, M.T., Yang, A., Albu, M., Cote, A.G., Montenegro-Montero, A., Drewe, P., Najafabadi, H.S., Lambert, S.A., Mann, I., Cook, K., et al. (2014). Determination and inference of eukaryotic transcription factor sequence specificity. Cell 158, 1431-1443.

Whitlock, M.C. (2005). Combining probability from independent tests: the weighted Z-method is superior to Fisher's approach. J Evol Biol 18, 1368-1373.

Yoh, S.M., Schneider, M., Seifried, J., Soonthornvacharin, S., Akleh, R.E., Olivieri, K.C., De Jesus, P.D., Ruan, C., de Castro, E., Ruiz, P.A., et al. (2015). PQBP1 Is a Proximal Sensor of the cGAS-Dependent Innate Response to HIV-1. Cell 161, 1293-1305.

York, A.G., Williams, K.J., Argus, J.P., Zhou, Q.D., Brar, G., Vergnes, L., Gray, E.E., Zhen, A., Wu, N.C., Yamada, D.H., et al. (2015). Limiting Cholesterol Biosynthetic Flux Spontaneously Engages Type I IFN Signaling. Cell 163, 1716-1729. 